\title{
A dam-break driven by a moving source: a simple model for a powder snow avalanche
}

\begin{tabular}{|c|c|}
\hline Journal: & Journal of Fluid Mechanics \\
\hline Manuscript ID & Draft \\
\hline mss type: & JFM Papers \\
\hline $\begin{array}{r}\text { Date Submitted by the } \\
\text { Author: }\end{array}$ & $n / a$ \\
\hline Complete List of Authors: & $\begin{array}{l}\text { Billingham, J; University of Nottingham, School of Mathematical } \\
\text { Sciences; School of Mathematical Sciences, University of Nottingham }\end{array}$ \\
\hline Keyword: & $\begin{array}{l}\text { Gravity currents < Geophysical and Geological Flows, Interfacial Flows } \\
\text { (free surface) }\end{array}$ \\
\hline
\end{tabular}

\section{SCHOLARONE ${ }^{m}$ Manuscripts}




\title{
A dam-break driven by a moving source: a simple model for a powder snow avalanche
}

\author{
John Billingham ${ }^{1} \dagger$ \\ ${ }^{1}$ School of Mathematical Sciences, The University of Nottingham, University Park, \\ Nottingham NG7 2RD, UK \\ (Received $\mathrm{xx}$; revised $\mathrm{xx}$; accepted $\mathrm{xx}$ )
}

We study the two-dimensional, irrotational flow of an inviscid, incompressible fluid injected from a line source moving at constant speed along a horizontal boundary, into a second, immiscible, inviscid fluid of lower density. A semi-infinite, horizontal layer sustained by the moving source has previously been studied as a simple model for a powder snow avalanche, Caroll et al. (2012). We show that with fluids of unequal densities, in a frame of reference moving with the source, no steady solution exists, and formulate an initial/boundary value problem that allows us to study the evolution of the flow. After considering the limit of small density difference, we study the fully nonlinear initial/boundary value problem and find that the flow at the head of the layer is effectively a dam-break for the initial conditions that we have used. We study the dynamics of this in detail for small times using the method of matched asymptotic expansions. Finally, we solve the fully nonlinear free boundary problem numerically using an adaptive vortex blob method, after regularising the flow by modifying the initial interface to include a thin layer of the denser fluid that extends to infinity ahead of the source. We find that the disturbance of the interface in the linear theory develops into a dispersive shock in the fully nonlinear initial/boundary value problem and overturns. For sufficiently large Richardson number, overturning can also occur at the head of the layer.

Key words: Avalanche, vortex sheet, vortex blob method, dam-break

\section{Introduction}

A powder snow avalanche is a flowing, fluidised suspension of fine snow, fed by new snow that erupts from the underlying surface due to large pressure gradients near the head of the avalanche, Sovilla et al. (2006); Louge et al. (2011). In Caroll et al. (2012), the authors consider a sequence of two-dimensional models of powder snow avalanches. In this paper we revisit the first of these, in which the fluidised snow is modelled as inviscid and incompressible, and is assumed to flow irrotationally from a moving point source surrounded by stationary, ambient inviscid fluid of lower density. When the fluid densities are equal, in a frame of reference moving at velocity $U>0$ with the point source of strength $q$ at its origin, the flow is given in polar coordinates by the velocity potential

$$
\phi=-U r \cos \theta+\frac{q}{\pi} \log r
$$

$\dagger$ Email address for correspondence: John.Billingham@Nottingham.ac.uk 


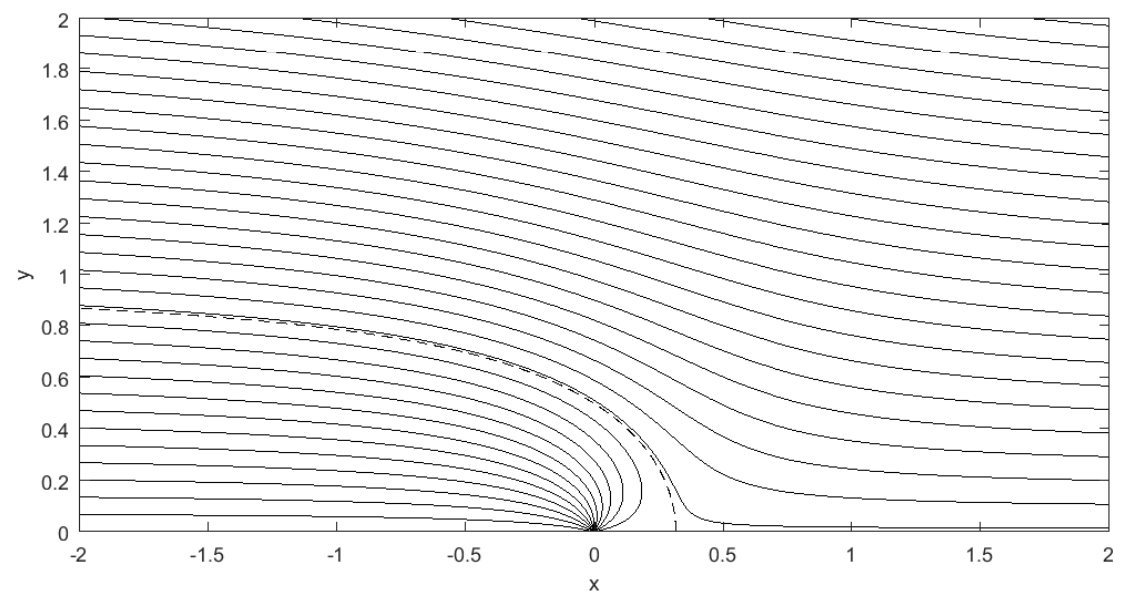

Figure 1. A unit source at $x=y=0$ in a unit flow from right to left when the two fluids have equal density, given by the velocity potential $\phi=-x+\frac{1}{\pi} \log r$ and corresponding streamfunction $\psi=-y+\frac{\theta}{\pi}$. The streamline that meets the stagnation point on the boundary at $x=1 / \pi, y=0$, and which represents the position of the free surface, $x=y \cot \pi y$ (equivalently $y=\theta / \pi$ ), is shown as a broken line.

in $0 \leqslant \theta \leqslant \pi$. Some typical streamlines are shown in Figure 1 and are equivalent to those around a Rankine body given by

$$
x=y \cot \left(\frac{\pi U y}{q}\right),
$$

in Cartesian coordinates (see, for example, Batchelor (1967)). In terms of the problem that we are interested in, $(1.2)$ is the dividing streamline between fluid 2 and fluid 1 , which originates at the source, when the fluids have equal density.

We find that the flow in this nonlinear, two fluid, free boundary problem is more complex than is suggested by the results presented in Caroll et al. (2012). Unless the fluids have equal density, no steady solution exists because of the likely nonexistence of a steady local solution in the neighbourhood of the contact point (at $y=0$ ). We analyse the dynamics of the flow by studying an initial value problem in which the initial velocity field is given by (1.1) and the fluids are initially separated by the free surface given by (1.2). Later in the paper, we also consider the flow when the initial position of the free surface is a nearby streamline of this flow, for reasons that will be become clear as we progress.

The difference in density between the fluids drives the subsequent development of the flow and motion of the free surface. Although it may seem more natural to study what happens when a source of slightly denser fluid is injected $a b$ initio, we are interested in 
how the dynamics of this flow are related to fully developed powder snow avalanches, for which the initial conditions that we have chosen are more relevant.

In Section 2, we set up the governing equations for this initial/boundary value problem. We study the problem in the limit of small density difference in Section3, and show that the head of the layer of fluid 1 (to the right of the source) swells and the contact point moves rapidly forwards close to the initial stagnation point. In addition, a disturbance propagates downstream at the speed of the source, which is a stationary initial transient in a frame of reference fixed in the boundary. In Section 4 we examine the initial behaviour of the flow, in particular near the stagnation point where the interface meets the solid surface. We find that this is locally a dam-break, which means that we need to use the method of matched asymptotic expansions, in the same manner as King \& Needham (1994) and Uddin \& Needham (2015), to determine the initial dynamics of the flow. Close to the contact point, we find that the free boundary takes a spatially oscillatory form, with a wavelength that tends to zero as $y \rightarrow 0$. We also find that in this region the Boussinesq approximation, $\bar{\rho} \ll 1$ and $\overline{\mathrm{Ri}}=O(1)$, which it is natural to consider in the outer flow (although it is not relevant to avalanches), is a singular limit. In Section 5 we discuss numerical solutions of the fully nonlinear free boundary problem, computed using an adaptive vortex blob method, which allows us to study the nonlinear development of the initial disturbance. We find that nonlinear effects (Kelvin-Helmholz instability) can cause the disturbance that propagates along the layer to overturn. For nonzero Richardson number, we regularise the flow close to the contact point by using an initial free surface with a thin layer of fluid 1 that extends to infinity ahead of the source. In this case, overturning can also occur at the head of the layer for sufficiently large Richardson number.

\section{Governing Equations}

Using the source velocity, $U$, and the flux from the source, $q$, we can form length and time scales, $L \equiv q / U$ and $T \equiv q / U^{2}$. We use these to non-dimensionalise the governing equations in a frame of reference moving with the source. The initial/boundary value problem that we will study, defined by 2.2 to $(2.8)$ below, contains just two dimensionless parameters constructed from these quantities: the dimensionless density difference and the Richardson number,

$$
\bar{\rho}=\frac{\rho_{1}-\rho_{2}}{\rho_{2}} \geqslant 0, \quad \mathrm{Ri}=\frac{\left(\rho_{1}-\rho_{2}\right) g q}{\rho_{2} U^{3}} \equiv \bar{\rho} \frac{g L}{U^{2}} \equiv \bar{\rho} \overline{\mathrm{Ri}} \geqslant 0 .
$$

In a powder snow avalanche, the density of the fluidised snow is typically between two and ten times greater than that of the surrounding air, the height of the avalanche is between $20 \mathrm{~m}$ and $50 \mathrm{~m}$, and typical propagation speeds lie between $15 \mathrm{~ms}^{-1}$ and $50 \mathrm{~ms}^{-1}$. This leads to the approximate parameter ranges $1 \leqslant \bar{\rho} \leqslant 10,0.1 \leqslant \mathrm{Ri} \leqslant 20$ and $0.1 \leqslant \overline{\mathrm{Ri}} \leqslant 2$. The Richardson number quantifies the importance of the effective gravitational force on the flow compared to that of the relative acceleration of the two fluids (the Bernoulli effect).

The two-dimensional domain of solution is $y \geqslant 0,-\infty<x<\infty$ in Cartesian coordinates. We denote by $\mathcal{D}_{i}(t)$ the region occupied by the fluid of density $\rho_{i}$ with velocity potential $\phi_{i}(\mathbf{x}, t)$, where $t \geqslant 0$ is time, $\mathbf{x}=(x, y)$ and $i=1$ or 2 . The boundary $\partial \mathcal{D}(t)$ between these two regions is given by $\mathbf{x}=\mathbf{X}(s, t) \equiv(X(s, t), Y(s, t))$, where $s$ is arc-length along the free surface, with $s=0$ at the point $x=X_{c}(t)$ where $\partial \mathcal{D}$ meets the boundary, $y=0$. The irrotational flow of the two incompressible, inviscid fluids is 
governed by

$$
\nabla^{2} \phi_{i}=0 \text { in } \mathcal{D}_{i}
$$

with the kinematic boundary condition

$$
\mathbf{n} \cdot \frac{\partial \mathbf{X}}{\partial t}=\mathbf{n} \cdot \nabla \phi_{1}=\mathbf{n} \cdot \nabla \phi_{2} \text { at } \mathbf{x}=\mathbf{X}(s, t),
$$

where $\mathbf{n}$ is the outward unit normal to $\mathcal{D}_{1}$, along with the dynamic boundary condition,

$$
(1+\bar{\rho})\left(\frac{\partial \phi_{1}}{\partial t}+\frac{1}{2}\left|\nabla \phi_{1}\right|^{2}\right)-\left(\frac{\partial \phi_{2}}{\partial t}+\frac{1}{2}\left|\nabla \phi_{2}\right|^{2}\right)=-\operatorname{Ri} Y \text { at } \mathbf{x}=\mathbf{X}(s, t) .
$$

Note that we have set the Bernoulli constant to zero, but that this is somewhat arbitrary, since the interface does not extend to infinity upstream. The no penetration condition at the solid boundary is

$$
\frac{\partial \phi_{1}}{\partial y}=\frac{\partial \phi_{2}}{\partial y}=0 \text { at } y=0 .
$$

Although 2.3 shows that the normal derivatives of the potentials, and hence normal velocities, are continuous at the boundary, the potentials and their tangential derivatives (the tangential velocities) need not be continuous in this inviscid model. We must also include the source of fluid 1 at the origin,

$$
\phi_{1} \sim \frac{1}{\pi} \log r \text { as } r \equiv \sqrt{x^{2}+y^{2}} \rightarrow 0,
$$

and the upstream flow of fluid 2 in this frame of reference,

$$
\phi_{2} \sim-x \text { as } x \rightarrow \infty
$$

Finally, we take the initial position of the free surface to be the Rankine surface for the flow with equal densities, given by 1.2 , so that

$$
X=Y \cot \pi Y \text { when } t=0,
$$

and hence when $t=0, \mathcal{D}_{1}$ is the region $0 \leqslant y \leqslant Y$ for $x \leqslant 1 / \pi$, with $\mathcal{D}_{2}$ the remainder of the upper half plane. Note that in Sections 3 and 4 , we will usually assume (correctly) that the free surface can be written as a single-valued function in polar coordinates, $r=R(\theta, t)$, with corresponding initial condition $R(\theta, 0)=\theta / \pi \sin \theta$, or as $y=Y(x, t)$ with $x=Y(x, 0) \cot \pi Y(x, 0)$ or as $x=X(y, t)$, with $X(y, 0)=y \cot \pi y$, in Cartesian coordinates. In Section 5, we will use a Lagrangian representation of the free surface, which can accommodate overturning, as part of our numerical solution method for the full initial/boundary value problem.

Although $\phi_{1}=\phi_{2}$ when $t=0$, as the inviscid, irrotational flow develops, a discontinuity in the tangential derivatives of these potentials, and hence a tangential velocity discontinuity, develops at the free surface. Locally, this will resemble planar, inviscid, two fluid flow with a velocity discontinuity. Flows like this are known to be subject to Kelvin-Helmholz instability (for example, Batchelor (1967)), which usually leads to the development of a curvature singularity in finite time (for example, Moore (1978), Cowley et al. (1999)), which can be regularised by adding some extra physics to the model, usually surface tension, although this is not the physics that we will add in this paper. We will address this issue in detail in Section 5. 


\section{Asymptotic solution for flows with small density difference $(\bar{\rho} \ll 1$, $\overline{\mathbf{R i}}=O(1)$ - the Boussinesq approximation)}

Although we have seen that $\bar{\rho}$ is not small for powder snow avalanches, it is natural to consider the Boussinesq limit, $\bar{\rho} \ll 1$ with $\overline{\mathrm{Ri}}=O(1)$, as a way to gain insight into the dynamics of the flow and provide a test for numerical solutions of the full problem. As we shall see, in this limit the head of the layer swells and a depression propagates back along the layer at unit speed. We will see in Section 5 that this is also how the flow behaves when $\bar{\rho}$ is not small. We will also discover that there is a singularity in the shape of the free surface close to the contact point (a dam-break), which persists when $\bar{\rho}$ is not small.

When $\bar{\rho} \ll 1$ and $\overline{\mathrm{Ri}}=O(1)$, it is most convenient to work in terms of polar coordinates and expand

$$
R(\theta, t)=R_{0}(\theta)+\bar{\rho} \bar{R}(\theta, t)+o(\bar{\rho}), \quad \phi_{i}(r, \theta, t)=\phi_{0}(r, \theta)+\bar{\rho} \bar{\phi}_{i}(r, \theta, t)+o(\bar{\rho}),
$$

where

$$
R_{0}(\theta) \equiv \frac{\theta}{\pi \sin \theta}, \quad \phi_{0} \equiv-r \cos \theta+\frac{1}{\pi} \log r .
$$

We begin by studying the dynamics of the vortex sheet strength, which decouples from the rest of the flow in this limit.

\subsection{The vortex sheet strength}

Note that, since 2.3 includes continuity of normal velocity, $\mathbf{n} \cdot \nabla \phi_{1}=\mathbf{n} \cdot \nabla \phi_{2}$ at $r=R(\theta, t)$, the dynamic boundary condition 2.4 at $O(\bar{\rho})$ is

$$
\frac{\partial \bar{\phi}_{1}}{\partial t}-\frac{\partial \bar{\phi}_{2}}{\partial t}+\frac{1}{2}\left(\mathbf{t} \cdot \nabla \phi_{0}\right)^{2}+\left(\mathbf{t} \cdot \nabla \phi_{0}\right)\left(\mathbf{t} \cdot \nabla \bar{\phi}_{1}-\mathbf{t} \cdot \nabla \bar{\phi}_{2}\right)=-\overline{\operatorname{Ri}} R_{0} \sin \theta \text { at } r=R_{0}(\theta),
$$

where $\mathbf{t}(s)$ is the unit tangent to the boundary. If we now define

$$
\mu(\theta, t) \equiv \bar{\phi}_{1}\left(R_{0}(\theta), \theta, t\right)-\bar{\phi}_{2}\left(R_{0}(\theta), \theta, t\right)
$$

(3.3) becomes

$$
\frac{\partial \mu}{\partial t}+\left(\mathbf{t} \cdot \nabla \phi_{0}\right) \frac{\partial \mu}{\partial s}=-\overline{\operatorname{Ri}} R_{0} \sin \theta-\frac{1}{2}\left(\mathbf{t} \cdot \nabla \phi_{0}\right)^{2} \quad \text { at } r=R_{0}(\theta),
$$

and hence, using the known functional forms of $R_{0}(\theta)$ and $\phi_{0}(r, \theta)$,

$$
\frac{\partial \mu}{\partial t}+\frac{\pi \sin ^{2} \theta}{\theta} \frac{\partial \mu}{\partial \theta}=-\overline{\mathrm{Ri}} \frac{\theta}{\pi}-\frac{\sin ^{2} \theta-2 \theta \sin \theta \cos \theta+\theta^{2}}{2 \theta^{2}}
$$

This semilinear equation determines the potential discontinuity across the free surface, and hence the jump in tangential velocity, or vortex sheet strength.

The steady solution of 3.4 is

$$
\mu=\mu_{0}(\theta) \equiv \mu_{0}(0)-\frac{\overline{\mathrm{Ri}}}{\pi^{2}} \int_{0}^{\theta} \frac{\alpha^{2}}{\sin ^{2} \alpha} d \alpha+\frac{1}{2 \pi}\left\{\theta \cot \theta-1+\log \left(\frac{\sin \theta}{\theta}\right)\right\},
$$

which is shown for various values of Ri in Figure 2, We find that

$$
\mu_{0} \sim-\left(\overline{\mathrm{Ri}}+\frac{1}{2}\right) \frac{1}{\pi-\theta}-\frac{1}{\pi}\left(2 \overline{\mathrm{Ri}}-\frac{1}{2}\right) \log (\pi-\theta) \quad \text { as } \theta \rightarrow \pi .
$$



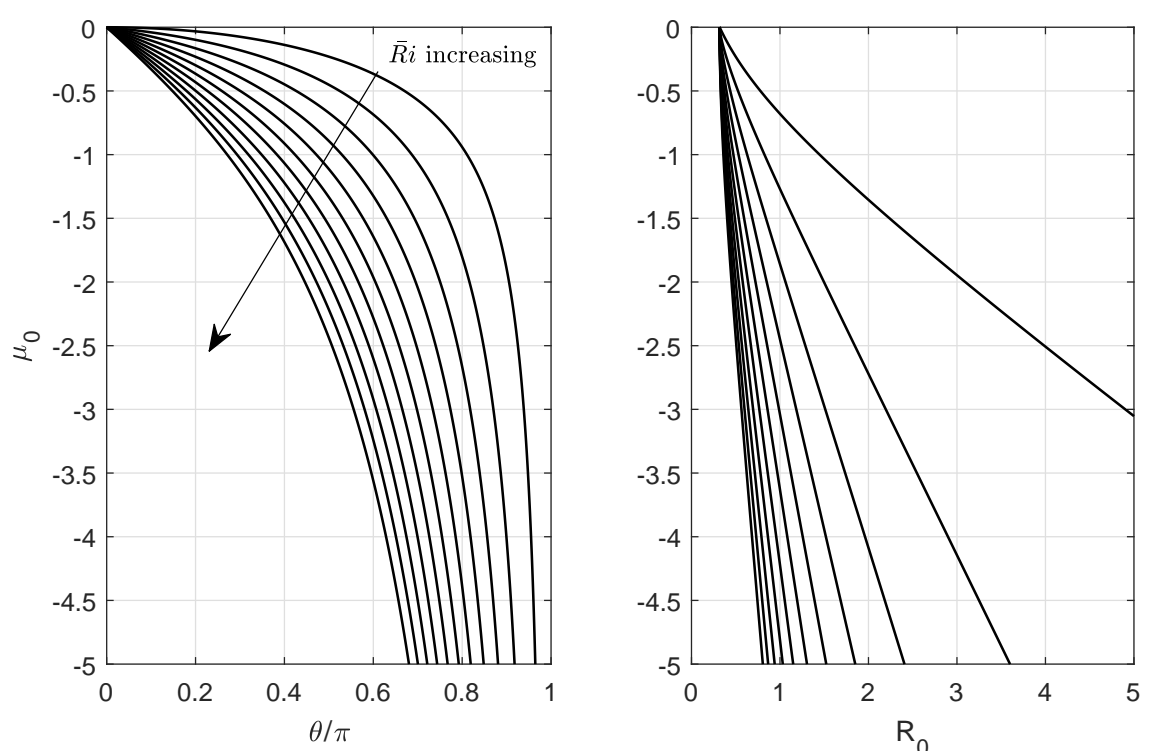

Figure 2 . The steady solution, $\mu_{0}$, for $\overline{\mathrm{Ri}}=0,1,2 \ldots, 10$, as a function of $\theta$ (left) and $R_{0}$ (right).

Since $R_{0} \sim(\pi-\theta)^{-1}$ as $\theta \rightarrow \pi$, we can write this as

$$
\mu_{0} \sim-\left(\overline{\mathrm{Ri}}+\frac{1}{2}\right) r+\frac{1}{\pi}\left(2 \overline{\mathrm{Ri}}-\frac{1}{2}\right) \log r \text { as } r \rightarrow \infty,
$$

which shows that the jump in tangential velocity tends to $\overline{\mathrm{Ri}}+\frac{1}{2}$ downstream, as $x \rightarrow$ $-\infty$.

In order to understand the unsteady dynamics of (3.4), note that the solution develops on characteristic curves given by

$$
\log \sin \theta-\theta \cot \theta=\pi t+\log \sin \theta_{0}-\theta_{0} \cot \theta_{0}
$$

which pass through $\theta=\theta_{0}$ when $t=0$, as shown in Figure 3 . Since $R_{0} \sim 1 /(\pi-\theta)$ and $d \theta / d t \sim(\pi-\theta)^{2}$ as $\theta \rightarrow \pi$, we find that, in terms of $R_{0}$, the characteristics have $d R_{0} / d t \rightarrow 1$ as $R_{0} \rightarrow \infty$, as shown in Figure 4 Physically, this means that, away from what we can see in Figure 4 is a small neighbourhood of the head of the region occupied by fluid 1 (at $x=1 / \pi, y=0$ ), disturbances propagate along the interface at unit speed, which is the dimensionless speed of the source. The closer to $x=1 / \pi$ the characteristic originates, the longer it remains in its neighbourhood before escaping to infinity, with $\theta_{0} \sim \theta e^{-\pi t}$ as $\theta \rightarrow 0$.

The solution of (3.4) is then given by

$$
\mu(\theta, t)=\mu\left(\theta_{0}, 0\right)-\frac{\overline{\mathrm{Ri}}}{\pi^{2}} \int_{\theta_{0}}^{\theta} \frac{\alpha^{2}}{\sin ^{2} \alpha} d \alpha+\frac{1}{2 \pi}\left\{\theta \cot \theta-\theta_{0} \cot \theta_{0}+\log \left(\frac{\theta_{0} \sin \theta}{\theta \sin \theta_{0}}\right)\right\} .
$$

The solution with initial condition $\mu(\theta, 0)=0$ is shown in Figure 5 for various values of $\overline{\mathrm{Ri}}$. It is straightforward to evaluate this accurately for any given values of $\theta$ and $t$ by solving (3.7) numerically to find $\theta_{0}$ and then evaluating (3.8) numerically. The solution can be seen to have two distinct regions: $0 \leqslant \theta<\theta_{i}(t)$, in which the solution is given by 


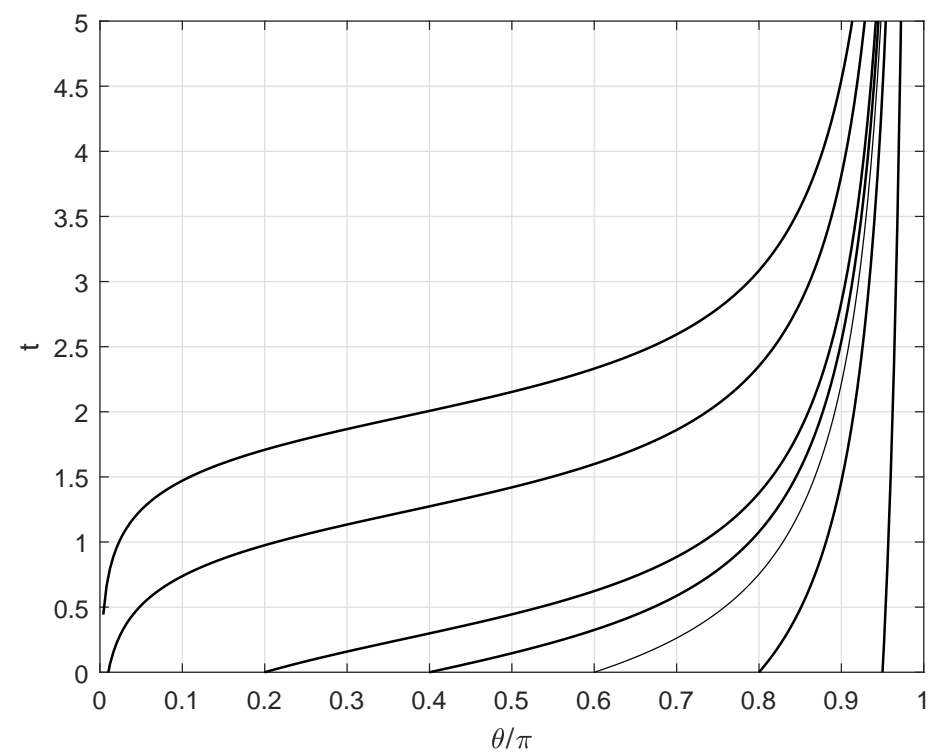

FIgURE 3. Some characteristic curves of the semilinear equation (3.4).

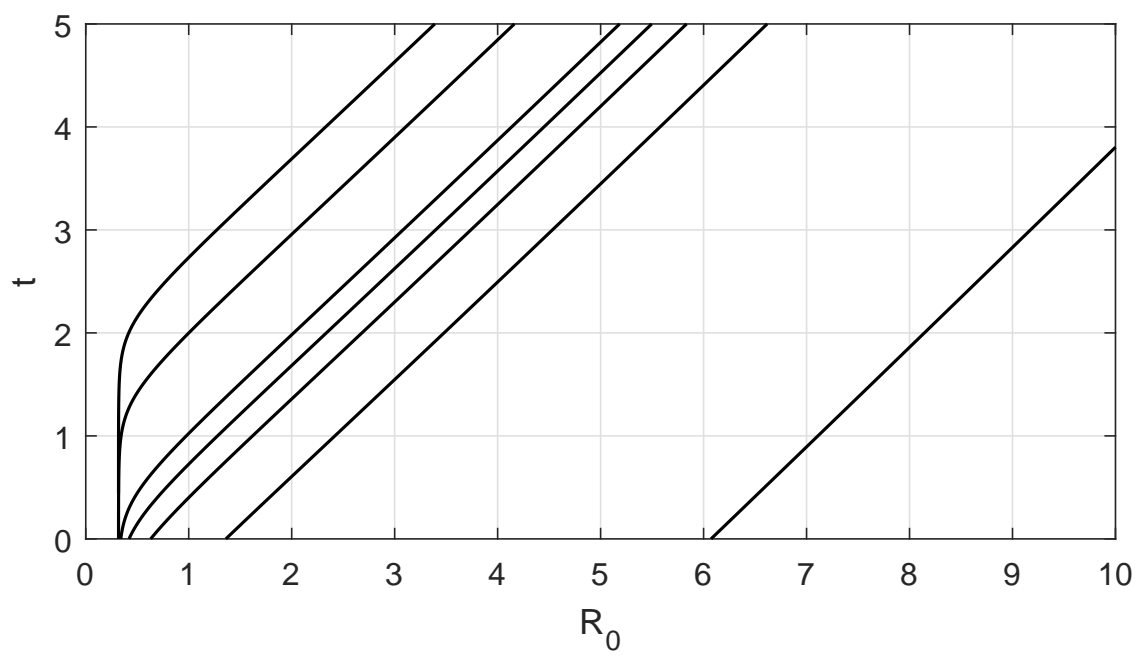

FiguRE 4. The characteristic curves of the semilinear equation (3.4 shown in Figure 3 redrawn in terms of $R_{0}(\theta)$. Note that $R_{0}(\theta)$ is a monotonically increasing function with $R_{0}(0)=1 / \pi$. 

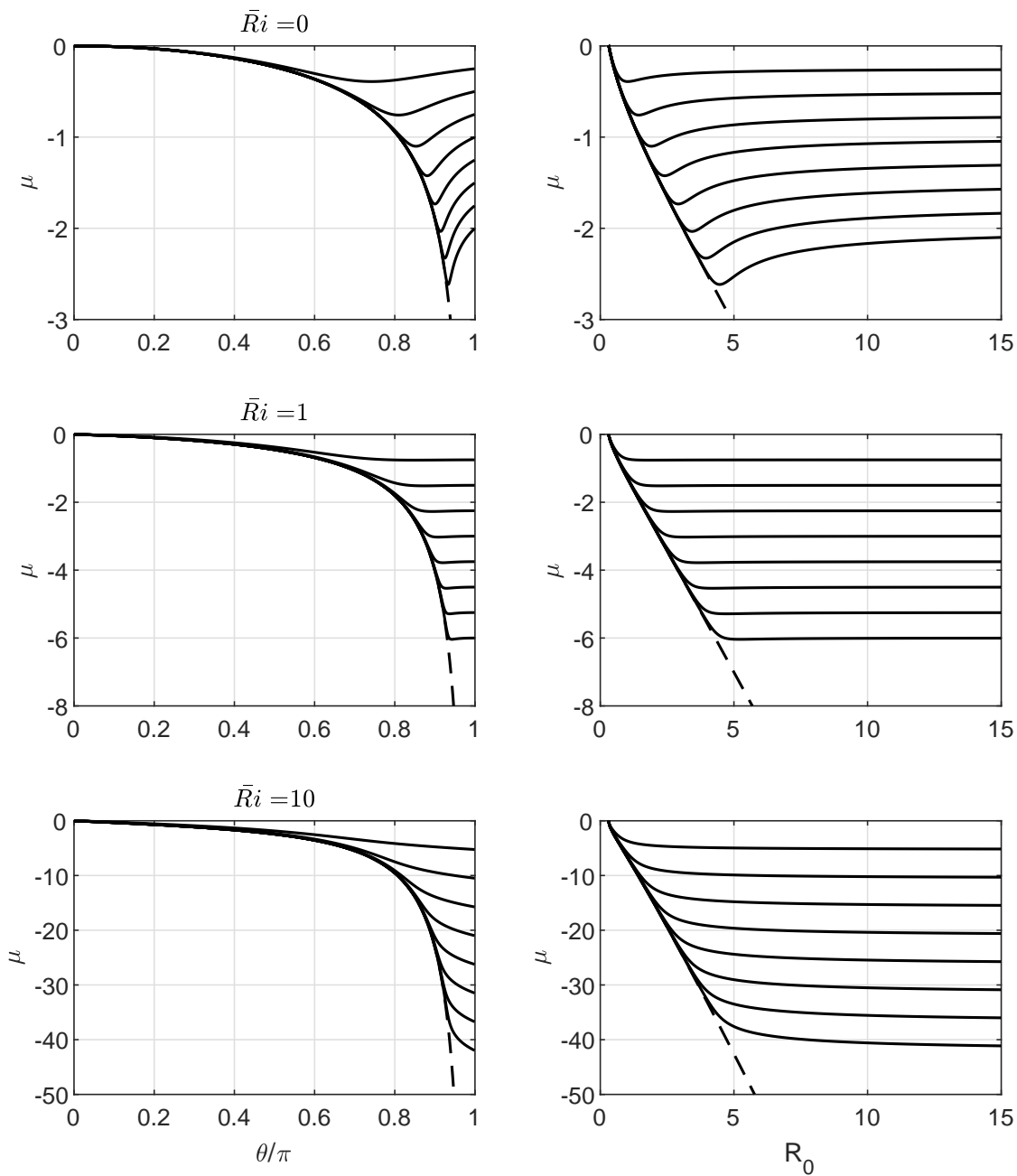

Figure 5. The solution, $\mu(\theta, t)$, when $\mu(\theta, 0)=0$ and $t=0.5,1,1.5,2,2.5,3,3.5$ and 4 , plotted as a function of $\theta$ (left) and $R_{0}$ (right), for $\mathrm{Ri}=0,1$ and 10 . The steady state solution is shown as a broken line.

the steady form $\mu=\mu_{0}(\theta)$, and $\theta_{i}(t)<\theta \leqslant \pi$, in which the solution grows with $t$. We can confirm this analytically by considering the large time form of the solution.

\subsection{Solution for $t \gg 1$}

It is clear from (3.7) that when $t \gg 1, \theta_{0} \ll 1$ and $\mu \sim \mu_{0}$, unless $\theta$ is close to $\pi$. By considering the forms of 3.5 and 3.7 when $\pi-\theta=O\left(t^{-1}\right)$, we find that the asymptotic solution does indeed consist of two distinct regions, separated by a thin, interior layer.

In summary, as $t \rightarrow \infty$,

$$
\theta_{i} \sim \pi-\frac{1}{t}-\frac{(\log t-1)}{\pi t^{2}}
$$


Region I: $0 \leqslant \theta<\theta_{i}-O\left(t^{-2}\right)$.

$$
\mu=\mu_{0}(\theta)+o(1)
$$

Region II: $\left|\theta-\theta_{i}\right|=O\left(t^{-2}\right)$.

$$
\begin{gathered}
\theta \sim \theta_{i}+\frac{1}{\pi t^{2}}\left(1+\log \sin \theta_{0}-\theta_{0} \cot \theta_{0}\right), \\
\mu \sim-\frac{\overline{\mathrm{Ri}}}{\pi^{2}} \int_{\theta_{0}}^{\theta_{i}} \frac{\alpha^{2}}{\sin ^{2} \alpha} d \alpha+\frac{1}{2 \pi}\left\{\theta \cot \theta-\theta_{0} \cot \theta_{0}+\log \left(\frac{\theta_{0} \sin \theta}{\theta \sin \theta_{0}}\right)\right\} .
\end{gathered}
$$

Region III: $\theta_{i}+O\left(t^{-2}\right)<\theta \leqslant \pi$.

$$
\mu \sim-\left(\overline{\mathrm{Ri}}+\frac{1}{2}\right) t-\frac{(\overline{\mathrm{Ri}}-1)}{\pi} \log \{1-t(\pi-\theta)\} .
$$

Note that the solution in Region III shows that the sign of $d \mu / d \theta$ changes from negative to positive as $\overline{\mathrm{Ri}}-1$ increases past zero when $t \gg 1$, which can clearly be seen in Figure 5 , and indicates a change in the nature of the solution in the far field.

The asymptotic solution for $t \gg 1$ is in excellent agreement with the exact solution, even for low values of $t$. The solution when $t=4$ and $\mathrm{Ri}=10$ is shown in Figure 6, along with the asymptotic solution in Regions I and III. Far downstream, as $r \rightarrow \infty$,

$$
\mu \sim-\left(\overline{\mathrm{Ri}}+\frac{1}{2}+\frac{\overline{\mathrm{R}} \mathrm{i}-1}{\pi R_{0}}\right) t .
$$

This shows that the asymptotic expansion 3.1 becomes nonuniform when $t=O\left(\bar{\rho}^{-1}\right)$ when $\bar{\rho} \ll 1$, and we expect that the solution becomes nonlinear on this timescale. We will discuss this further in Section 5 .

\subsection{The normal velocity at the free surface, $\mathbf{n} \cdot \nabla \bar{\phi}_{1}=\mathbf{n} \cdot \nabla \bar{\phi}_{2}$}

In order to determine the normal velocity at the free surface, which controls its motion, we use the vortex sheet representation (see, for example, Baker et al. (1982)),

$$
\mathbf{n} \cdot \nabla \bar{\phi}_{i}(s, t)=\frac{1}{2 \pi} \mathbf{t}(s) \cdot f_{-\infty}^{\infty} \mu_{s}\left(\theta\left(s^{\prime}\right), t\right) \frac{\mathbf{X}(s)-\mathbf{X}\left(s^{\prime}\right)}{\left|\mathbf{X}(s)-\mathbf{X}\left(s^{\prime}\right)\right|^{2}} d s^{\prime},
$$

where $\mathbf{t}(s) \equiv\left(X_{s}(s), Y_{s}(s)\right)$ is the unit vector tangent to the boundary and $\mu_{s} \equiv \partial \mu / \partial s$ is the jump in the tangential velocity at the free surface, also known as the normalised vortex sheet strength. We have extended the free surface by reflection in the $x$-axis, with $\mu_{s}(-\theta, t)=\mu_{s}(\theta, t), X(-s)=X(s)$ and $Y(-s)=-Y(s)$ by symmetry. The arc-length along the fixed boundary can be calculated from

$$
\frac{d \theta}{d s}=\frac{\pi \sin ^{2} \theta}{\sqrt{\sin ^{2} \theta-2 \theta \sin \theta \cos \theta+\theta^{2}}} .
$$

Equation (3.10) allows us to determine the normal velocity from the vortex sheet strength, $\mu_{s}$, which we discussed above. Note that it is possible to use conformal mapping to find the individual velocity potentials, $\bar{\phi}_{1}$ and $\bar{\phi}_{2}$, as integrals of $\mathbf{n} \cdot \nabla \bar{\phi}_{i}$, but we will not present any details here as it is possible to determine the motion of the free surface from the normal velocity alone.

Figure 7 shows how the vortex sheet strength varies for the same values of time, $t$, used in Figure 5. We can see that $\mu_{s}$ approaches the steady state solution 


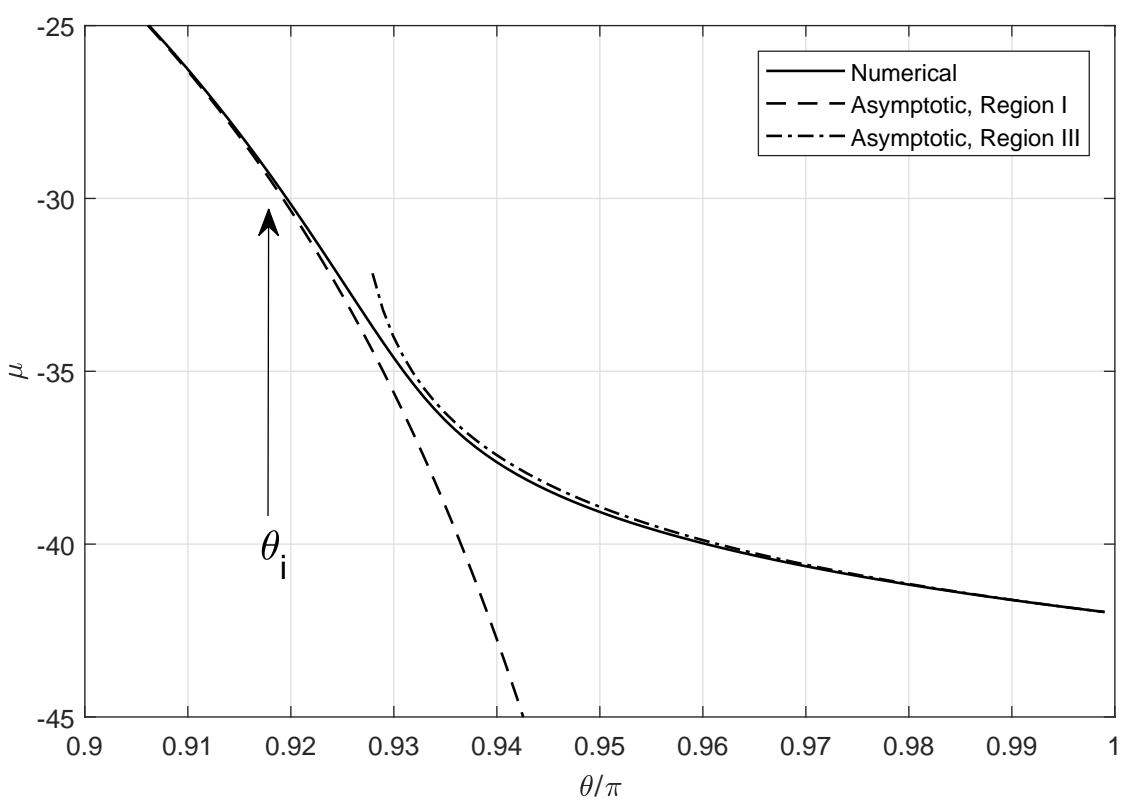

Figure 6 . The solution, $\mu(\theta, t)$, when $\mu(\theta, 0)=0, \overline{\mathrm{Ri}}=10$ and $t=4$, along with the asymptotic solution for $t \gg 1$ in Regions I and III. The position of the interior layer, at $\theta=\theta_{i}$, is also indicated. The value of $\theta_{i}$ is not very accurate for this low value of $t$, but for sufficiently large values of $t$ the asymptotic and numerical solutions become indistinguishable.

$$
\mu_{0 s}(\theta)=-\frac{\overline{\mathrm{Ri}} \theta^{2}}{\pi \sqrt{\sin ^{2} \theta-2 \theta \sin \theta \cos \theta+\theta^{2}}}-\frac{\sqrt{\sin ^{2} \theta-2 \theta \sin \theta \cos \theta+\theta^{2}}}{2 \theta},
$$

in Region I as $t$ increases, following the asymptotic solution described in Section 3.2 even at moderate times. In asymptotic Region III, where $R>t-\frac{1}{\pi}(\log t-1)+O\left(t^{-1}\right)$,

$$
\mu_{s} \sim-\frac{(\overline{\mathrm{Ri}}-1) t}{R(R-t)} \text { as } t \rightarrow \infty .
$$

In order to determine the behaviour of the free surface, we use the known functional forms of $\phi_{0}(r, \theta)$ and $R_{0}(\theta)$ to show that the radial perturbation of the free surface satisfies

$$
\frac{\partial \bar{R}}{\partial t}+\frac{\pi \sin ^{2} \theta}{\theta} \frac{\partial \bar{R}}{\partial \theta}=-\frac{\pi \sin \theta \cos \theta}{\theta} \bar{R}+\frac{\sqrt{\sin ^{2} \theta-2 \theta \sin \theta \cos \theta+\theta^{2}}}{\theta \sin \theta} \mathbf{n} \cdot \nabla \bar{\phi}_{i} \quad \text { at } r=R_{0}(\theta) .
$$

In order to solve the simple, linear equation 3.12 numerically, we discretise in $\theta$ and approximate $\partial \bar{R} / \partial \theta$ using first order, upwind finite differences. The resulting system of ordinary differential equations can then be solved using ode 45 in MATLAB, coupled with the simple initial value problem for $\theta_{0}(t ; \theta)$,

$$
\frac{d \theta_{0}}{d t}=-\frac{\pi \sin ^{2} \theta_{0}}{\theta_{0}}, \text { subject to } \theta_{0}(0 ; \theta)=\theta .
$$

This follows from integrating back along a characteristic at fixed $\theta$, and allows $\theta_{0}$, and hence $\mu_{s}$, to be calculated efficiently as part of the solution. The principal value integral in 3.10 can be evaluated numerically, working in terms of $\theta$, subtracting out the singular behaviour in the neighbourhood of $s=s^{\prime}\left(\theta=\theta^{\prime}\right)$ and using four point 

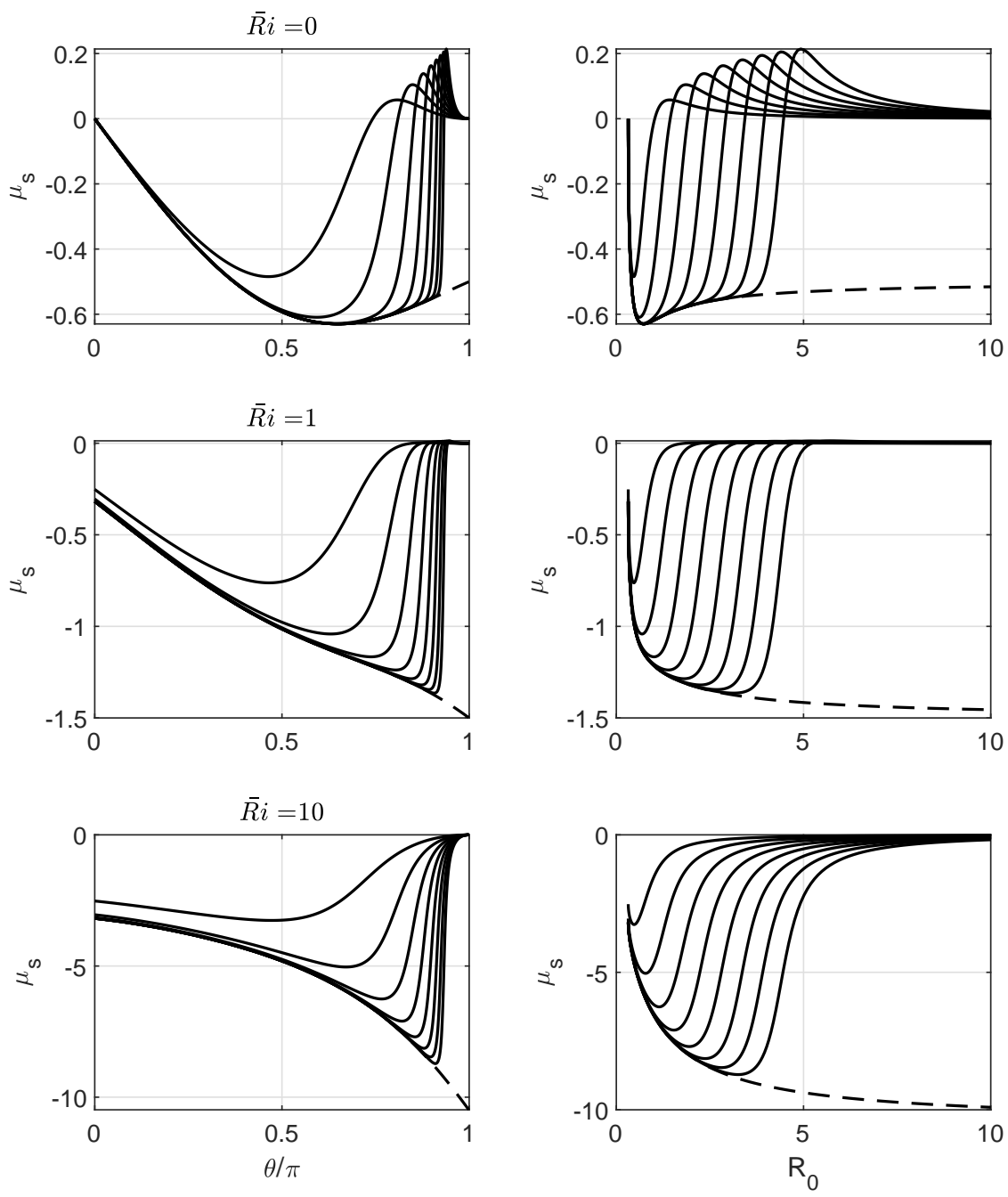

FiguRE 7 . The normalised vortex sheet strength, $\mu_{s}$, when $\mu(\theta, 0)=0$ and $t=0.5,1,1.5,2$, $2.5,3,3.5$ and 4 , the times that are used in Figure 5 for $\mathrm{Ri}=0,1$ and 10 . The broken line shows the steady solution, $\mu_{0 s}$, in each case.

Gaussian quadrature. This linear relationship means that the discretised version of $\mathbf{n} \cdot \nabla \phi_{i}$ can be found from $\mu_{s}\left(\theta ; \theta_{0}(t)\right)$ by multiplication by a constant matrix, which can be precalculated.

Figure 8 shows the position of the free boundary when $t=1$ for $\bar{\rho}=0.1$ and various values of $\mathrm{Ri}$. This somewhat large value of $\bar{\rho}$ has been chosen to allow the position of the interface to be seen more clearly. In each case, the layer swells at the head, with the size of the swelling increasing with $\overline{\mathrm{Ri}}$ and $\bar{\rho}$, whilst a depression propagates back down the layer at unit speed. Note that when $\mathrm{R} i=0$ (the zero gravity case), the free surface remains perpendicular to the boundary, $y=0$, and the speed of the contact point asymptotes to zero as $t \rightarrow \infty$. When $\overline{\mathrm{Ri}}>0$, a thin layer of fluid 1 protrudes into fluid 2 


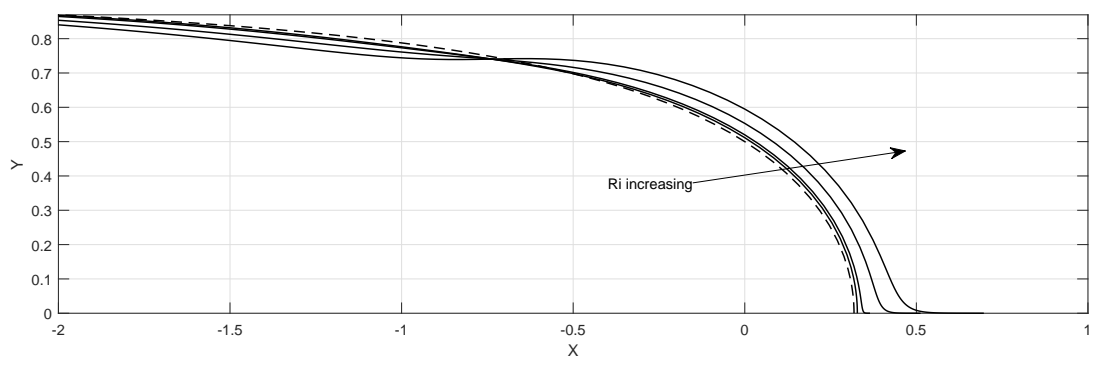

Figure 8 . The position of the free surface for $\bar{\rho}=0.1$ and $\overline{\mathrm{Ri}}=0,1,5$ and 10 when $t=1$, calculated from the asymptotic solution for $\bar{\rho} \ll 1$. The initial position of the free surface is shown as a broken line.

close to the boundary, with the shape of the free surface very different to that in the zero gravity case. This is because there is a logarithmic singularity in the position of the free surface as $y \rightarrow 0$, which we have not dealt with explicitly in our evaluation of the integral in 3.10 , and is therefore removed by rounding error when $y=0$. The behaviour close to the boundary when $\mathrm{Ri}=O(1)$ and $t \ll 1$ is the subject of Section 4 , and includes the limit $\bar{\rho} \ll 1$, which we have studied in this Section, as a subcase. In order to see why there is a singularity, consider the dynamic boundary condition, 2.4. Initially, the free surface is perpendicular to the boundary, $y=0$, so we can differentiate (2.4) with respect to $y$, and use the no penetration condition, (2.5), to show that the left hand side is then zero. The right hand side is, however, nonzero, and it is this incompatibility in the boundary conditions that leads to a singularity in the outer flow when $t \ll 1$. This incompatibility is reconciled in a small inner region close to the point of contact of the free surface with the boundary which, crucially, moves with an algebraically-small velocity and a logarithmically-large acceleration as $t \rightarrow 0$. In Section 5 we determine an equation for the motion of the contact point, (5.7), that holds for all $t>0$.

Figure 9 shows the free surface at later times for various values of $\overline{\mathrm{Ri}}$ and $\bar{\rho}$. The depression of the free boundary can be seen to continue to propagate at unit speed whilst deepening, steepening and eventually overturning (although the position of the free boundary remains a single-valued function $\left.r=R_{0}(\theta)+\bar{\rho} \bar{R}(\theta, t)\right)$. The larger the value of $\overline{\mathrm{Ri}}$, the deeper the depression (note the different choices of $\bar{\rho}$ in each panel of Figure 9). As the depression deepens and the slope of the boundary increases, this linear theory breaks down. As we shall see in Section 5 nonlinearity leads to a dispersive shock at the rear of the depression (see Figure 18), and the eventual formation of a singularity in the curvature. Ahead of the depression, the free boundary asymptotes to a locally steady solution with thickness larger than that of the original layer. Note however that, as discussed above, for $\overline{\mathrm{Ri}}>0$, the position of the free surface is logarithmically singular as $y \rightarrow 0$. The bounded solution computed in this outer region is an artefact of the method that we have used to approximate the integral in 3.10 , with rounding error 

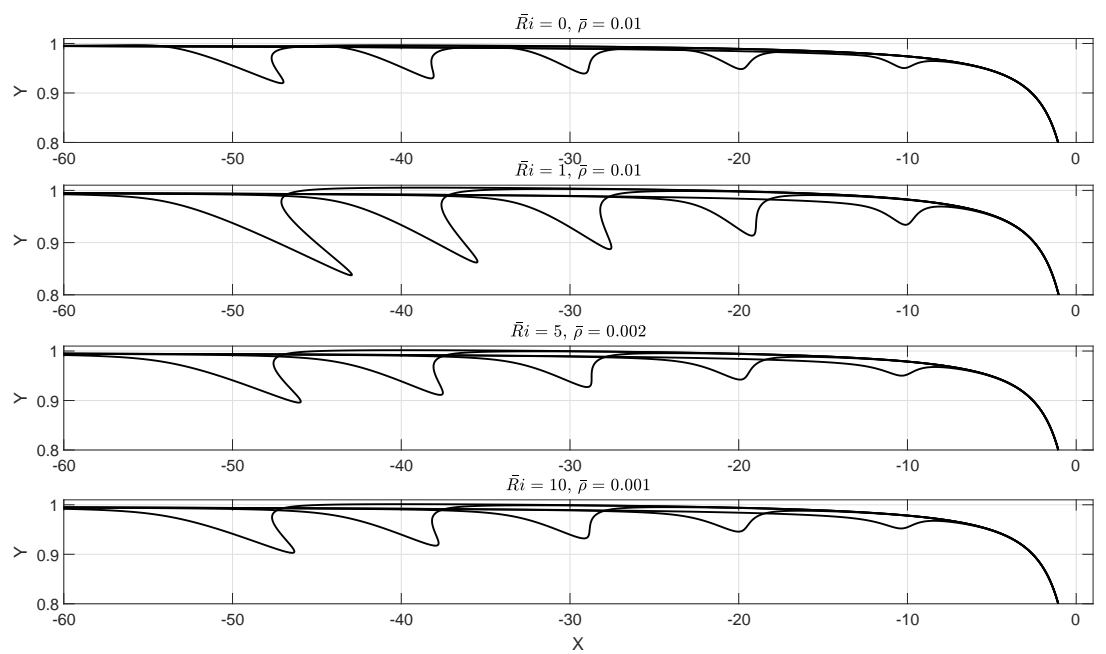

Figure 9 . The position of the free surface when $t=10,20,30,40$ and 50 for various values of $\overline{\mathrm{Ri}}$ and $\bar{\rho}$, calculated from the asymptotic solution for $\bar{\rho} \ll 1$. The values of $\bar{\rho}$ are chosen to give deformations of the surface that are neither too small to see clearly, nor too large. Note the unequal scales of the axes.

cutting off the singularity. This indicates how easy it is to compute spurious numerical solutions for this problem, since the logarithmic singularity is so weak.

\section{The dam-break solution when $t \ll 1, \bar{\rho}=O(1)$ and $\mathbf{R i}=O(1)$}

The solution when $t \ll 1$ with $\bar{\rho}=O(1)$ and $\mathrm{Ri}=O(1)$ consists of two asymptotic regions: an outer region, where the solution is a small perturbation of the initial conditions, and an inner region, close to the point of contact of the free surface with the boundary, $y=0$, where the logarithmic singularity in the outer flow is resolved. In each region, it is most convenient to represent the free surface as $x=X(y, t)$. Note that for this problem, we will show that the Boussinesq approximation $(\bar{\rho}=0$ with $\mathrm{Ri}>0)$ is a singular perturbation. The acceleration of the two fluids in the inner region is crucial in resolving the outer singularity, and if the two fluids are treated as having equal density in the inertial terms in (2.3), this acceleration is not associated with a restoring force. As noted earlier, the flow in the inner region is effectively a two-fluid dam-break, and we use the methods developed by King \& Needham (1994) and Uddin \& Needham (2015) to determine the asymptotic solution as $t \rightarrow 0$.

$$
\text { 4.1. Outer solution: } \phi_{i}=\phi_{0}+O(t), X=X_{0}+O\left(t^{2}\right)
$$

We define scaled variables

$$
\phi_{i}=\phi_{0}(x, y)+t \bar{\phi}_{i}(x, y, t), \quad X=X_{0}(y)+t^{2} \bar{X}(y, t),
$$

with $\bar{\phi}_{i}, \bar{X}=O(1)$ as $t \rightarrow 0$. At leading order, we obtain the boundary value problem

$$
\nabla^{2} \bar{\phi}_{i}=0 \text { in } \mathcal{D}_{i}(0),
$$

subject to

$$
\frac{\partial \bar{\phi}_{i}}{\partial y}=0 \text { at } y=0
$$




$$
\begin{gathered}
(1+\bar{\rho}) \bar{\phi}_{1}-\bar{\phi}_{2}=-\operatorname{Ri} y-\frac{1}{2} \bar{\rho}\left|\nabla \phi_{0}\right|^{2} \text { at } x=X_{0}(y), \\
2 \bar{X}=\frac{\partial \bar{\phi}_{i}}{\partial x}-\frac{d X_{0}}{d y} \frac{\partial \bar{\phi}_{i}}{\partial y} \text { at } x=X_{0}(y) .
\end{gathered}
$$

Note that $\bar{X}(y)$ decouples from the outer problem, and can be determined from 4.4 once the normal velocity at the free surface is known.

\subsubsection{Local solution as $(x-1 / \pi)^{2}+y^{2} \rightarrow 0$}

Close to the point $x=1 / \pi, y=0$, where the undisturbed free surface meets the boundary (a stagnation point of the initial potential, $\phi_{0}(x, y)$ ), the incompatibility in the boundary conditions (4.2) and 4.3 leads to a logarithmic singularity, as discussed at the end of Section 3 . The local solution is

$$
\begin{aligned}
& \bar{\phi}_{1} \sim p_{0}-\frac{\mathrm{Ri}}{\pi\left(1+\frac{1}{2} \bar{\rho}\right)}\{r \log r \cos \theta+(\pi-\theta) r \sin \theta+k r \cos \theta\} \quad \text { as } r \rightarrow 0, \\
& \bar{\phi}_{2} \sim(1+\bar{\rho}) p_{0}-\frac{\mathrm{Ri}}{\pi\left(1+\frac{1}{2} \bar{\rho}\right)}\{r \log r \cos \theta-\theta r \sin \theta+k r \cos \theta\} \quad \text { as } r \rightarrow 0,
\end{aligned}
$$

where $(r, \theta)$ is here a set of polar coordinates centred on $(1 / \pi, 0)$, and $p_{0}$ and $k$ are parameters that are determined globally. Although $p_{0} \equiv p_{0}(\rho, \overline{\mathrm{Ri}})$ is unimportant, $k \equiv$ $k(\bar{\rho}, \mathrm{Ri})$ affects the solution in the inner region. By using (4.5) in (4.4), we find that

$$
\bar{X} \sim-\frac{\mathrm{Ri}}{2 \pi\left(1+\frac{1}{2} \bar{\rho}\right)}\left\{\log Y_{0}(s)+1+k\right\} \quad \text { as } s \rightarrow 0 .
$$

It is this logarithmic singularity in the position of the free surface in the outer solution that leads to an inner region where the singularity is resolved.

We will also use the streamfunctions, $\bar{\psi}_{1}$ and $\bar{\psi}_{2}$, that correspond to the velocity potentials $\bar{\phi}_{1}$ and $\bar{\phi}_{2}$, which have

$$
\begin{aligned}
\bar{\psi}_{1} \sim q-\frac{\mathrm{Ri}}{\pi\left(1+\frac{1}{2} \bar{\rho}\right)}\{r \log r \sin \theta-(\pi-\theta) r \cos \theta+k r \sin \theta\} & \text { as } r \rightarrow 0, \\
\bar{\psi}_{2} \sim q-\frac{\mathrm{Ri}}{\pi\left(1+\frac{1}{2} \bar{\rho}\right)}\{r \log r \sin \theta+\theta r \cos \theta+k r \sin \theta\} & \text { as } r \rightarrow 0 .
\end{aligned}
$$

We will use capital letters with subscripts to denote the potentials and streamfunctions on the boundary as a function of arc-length, $s$, and note that

$$
\begin{gathered}
\Phi_{1} \sim p_{0}-\frac{\mathrm{Ri}}{2\left(1+\frac{1}{2} \bar{\rho}\right)} Y_{0}(s), \quad \Phi_{2} \sim(1+\bar{\rho}) p_{0}+\frac{\mathrm{Ri}}{2\left(1+\frac{1}{2} \bar{\rho}\right)} Y_{0}(s), \\
\Psi_{1}=\Psi_{2} \equiv \Psi \sim q-\frac{\mathrm{Ri}}{\pi\left(1+\frac{1}{2} \bar{\rho}\right)}\left\{Y_{0}(s) \log Y_{0}(s)+k Y_{0}(s)\right\} \text { as } s \rightarrow 0 .
\end{gathered}
$$

\subsubsection{Solution using Plemelj formulae}

We define the complex potential, $\Phi(z)=\phi_{i}+i \psi_{i}$ in $\mathcal{D}_{i}$, with $z=x+i y$, which can be written in terms of the potential difference on the boundary, $\partial \mathcal{D}$, which we extend into the lower half plane by symmetry, as

$$
\Phi(z)=\frac{1}{2 \pi i} \int_{-\infty}^{\infty} \frac{\Phi_{1}(s)-\Phi_{2}(s)}{Z_{0}(s)-z} \frac{d Z_{0}}{d s}(s) d s,
$$


where $Z_{0}(s) \equiv X_{0}(s)+i Y_{0}(s)$. On the boundary, the Plemelj formulae (see for example, Ablowitz \& Fokas (1997)) show that

$$
\Phi_{1}(s)=\frac{1}{2}\left(\Phi_{1}(s)-\Phi_{2}(s)\right)+\Re\left[\frac{1}{2 \pi i} \int_{-\infty}^{\infty} \frac{\Phi_{1}\left(s^{\prime}\right)-\Phi_{2}\left(s^{\prime}\right)}{Z_{0}\left(s^{\prime}\right)-Z_{0}(s)} \frac{d Z_{0}}{d s}\left(s^{\prime}\right) d s^{\prime}\right] .
$$

We can now use 4.3 to eliminate $\Phi_{2}$, and obtain a single Fredholm integral equation of the second kind,

$\Phi_{1}(s)=-\frac{1}{1+\frac{1}{2} \bar{\rho}}\left\{\frac{1}{2} \operatorname{Ri}\left|Y_{0}(s)\right|-\Re\left[\frac{1}{2 \pi i} \int_{-\infty}^{\infty} \frac{\operatorname{Ri}\left|Y_{0}\left(s^{\prime}\right)\right|+\bar{\rho} \Phi_{1}\left(s^{\prime}\right)+\frac{1}{2} \bar{\rho}\left|\nabla \phi_{0}\right|^{2}}{Z_{0}\left(s^{\prime}\right)-Z_{0}(s)} \frac{d Z_{0}}{d s}\left(s^{\prime}\right) d s^{\prime}\right]\right\}$.

Note that the kernel is regular as $s^{\prime} \rightarrow s$, so this is not a principal value integral. When $\bar{\rho}=0$, this immediately gives $\Phi_{1}(s)$, and for $\bar{\rho}>0$ is in a form that can be solved iteratively, using four point Gaussian quadrature to evaluate the integral. In order to determine the displacement of the boundary, $\bar{X}$, note that the streamfunction on the boundary is

$$
\Psi(s)=\Im\left[-\frac{1}{2 \pi i} \int_{-\infty}^{\infty} \frac{\operatorname{Ri}\left|Y_{0}\left(s^{\prime}\right)\right|+\bar{\rho} \Phi_{1}\left(s^{\prime}\right)+\frac{1}{2} \bar{\rho}\left|\nabla \phi_{0}\right|^{2}}{Z_{0}\left(s^{\prime}\right)-Z_{0}(s)} \frac{d Z_{0}}{d s}\left(s^{\prime}\right) d s^{\prime}\right] .
$$

After regularising this integral and subtracting out the behaviour of $\Phi_{1}\left(s^{\prime}\right)$ close to $s^{\prime}=0$, which leads to the logarithmic behaviour of $\Psi$ as $s \rightarrow 0,4.8$, we can evaluate $\Psi$ using four point Gaussian quadrature. We can calculate $\partial \Psi / \partial s=\mathbf{n} \cdot \nabla \bar{\phi}_{i}$, and hence the displacement of the boundary, $\bar{X}$, using 4.4. Figure 10 shows the solution for various values of $\mathrm{Ri}$ and $\bar{\rho}$, although we have not plotted a point at $y=0$. As expected, there is a logarithmic singularity in $\bar{X}(y)$ as $y \rightarrow 0$, but it is too weak to be seen clearly in Figure 10. The head of fluid 1 swells and is displaced forwards close to the contact point, reproducing the behaviour that we saw for $\bar{\rho} \ll 1$ and $\overline{\mathrm{Ri}}=O(1)$ in Figure 8 . The rate of displacement and size of the swelling increases with $\bar{\rho}$ and $\mathrm{Ri}$, but is always qualitatively similar.

\subsection{Inner solution: $\phi_{i}=O\left(t^{3}(-\log t)^{2}\right), x-1 / \pi, y=O\left(t^{2}(-\log t)\right)$}

On comparing the size of the terms neglected in the outer region to those retained as $s \rightarrow 0$ (equivalently, $y \rightarrow 0$ on the boundary), we find that appropriate scaled variables are (see Uddin \& Needham (2015) for more details on the very similar, single fluid version of this dam-break problem)

$$
\begin{gathered}
x=\frac{1}{\pi}+\operatorname{Ri} t^{2}(-\log t) \hat{x}, \quad y=\operatorname{Ri} t^{2}(-\log t) \hat{y}, \quad X=\frac{1}{\pi}+\operatorname{Ri} t^{2}(-\log t) \hat{X}, \\
\phi_{i}=-\frac{1+\log \pi}{\pi}+\operatorname{Ri} t^{2}(1+(i-1) \bar{\rho}) p(\bar{\rho})+\operatorname{Ri}^{2} t^{3}(-\log t)^{2} \hat{\phi}_{i},
\end{gathered}
$$

with the hatted variables of $O(1)$ as $t \rightarrow 0$. In terms of these, the kinematic and Bernoulli equations, 2.3 and 2.4, become

$$
\begin{array}{r}
t \frac{\partial \hat{X}}{\partial t}+\left\{2-(-\log t)^{-1}\right\}\left(\hat{X}-\hat{y} \frac{\partial \hat{X}}{\partial \hat{y}}\right)=\frac{\partial \hat{\phi}_{i}}{\partial \hat{x}}-\frac{\partial \hat{X}}{\partial \hat{y}} \frac{\partial \hat{\phi}_{i}}{\partial \hat{y}} \text { at } \hat{x}=\hat{X} \\
t \frac{\partial}{\partial t}\left\{(1+\bar{\rho}) \hat{\phi}_{1}-\hat{\phi}_{2}\right\}+\left\{3-2(-\log t)^{-1}\right\}\left\{(1+\bar{\rho}) \hat{\phi}_{1}-\hat{\phi}_{2}\right\}
\end{array}
$$



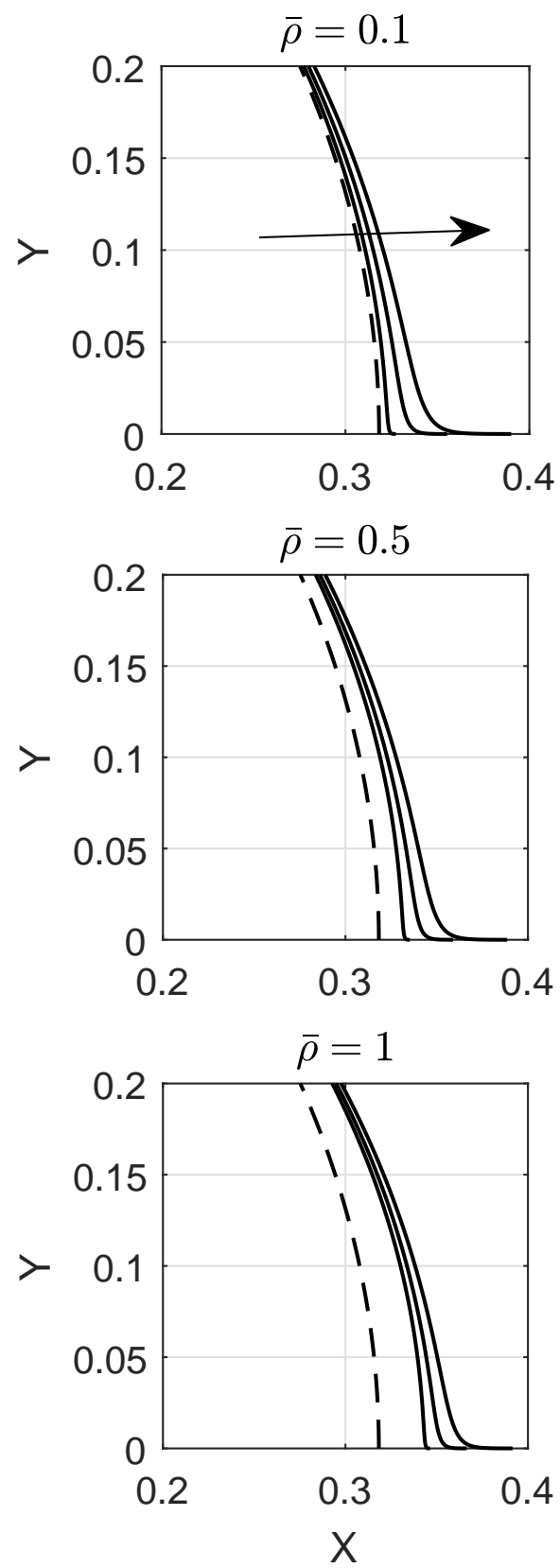

FiguRE 10. The boundary in the small time outer region when $\mathrm{Ri}=0.1,0.5$ and 1 with the values of $\bar{\rho}$ shown when $t=0.2$. The broken line shows the initial position of the boundary. Ri increases in the direction of the arrow. 


$$
\begin{gathered}
-\left\{2-(-\log t)^{-1}\right\} \hat{\mathbf{x}} \cdot \hat{\nabla}\left\{(1+\bar{\rho}) \hat{\phi}_{1}-\hat{\phi}_{2}\right\}+\frac{1}{2}\left\{(1+\bar{\rho})\left|\hat{\nabla} \hat{\phi}_{1}\right|^{2}-\left|\hat{\nabla} \hat{\phi}_{2}\right|^{2}\right\} \\
=-\hat{y}(-\log t)^{-1} \text { at } \hat{x}=\hat{X},
\end{gathered}
$$

and matching with the outer solution requires

$$
\begin{gathered}
\hat{\phi}_{1} \sim-\frac{1}{\pi\left(1+\frac{1}{2} \bar{\rho}\right)}[\hat{x}\{\log \hat{r}+k(\bar{\rho})+\log \mathrm{Ri}-2(-\log t)+\log (-\log t)\}+\hat{y}(\pi-\hat{\theta})] \text { as } \hat{r} \rightarrow \infty, \\
\hat{\phi}_{2} \sim-\frac{1}{\pi\left(1+\frac{1}{2} \bar{\rho}\right)}[\hat{x}\{\log \hat{r}+k(\bar{\rho})+\log \mathrm{Ri}-2(-\log t)+\log (-\log t)\}-\hat{y} \hat{\theta}] \quad \text { as } \hat{r} \rightarrow \infty, \\
\hat{X} \sim-\frac{1}{2 \pi\left(1+\frac{1}{2} \bar{\rho}\right)}\{\log \hat{y}+k(\bar{\rho})+1+\log \mathrm{Ri}-2(-\log t)+\log (-\log t)\} \quad \text { as } \hat{y} \rightarrow \infty .
\end{gathered}
$$

This suggests that we should expand the unknowns as

$$
\begin{aligned}
& \hat{\phi}_{i}=\hat{\phi}_{i 0}+\frac{\log (-\log t)}{-\log t} \hat{\phi}_{i 1}+\frac{1}{-\log t} \hat{\phi}_{i 2}+o\left((-\log t)^{-1}\right), \\
& \hat{X}=\hat{X}_{0}+\frac{\log (-\log t)}{-\log t} \hat{X}_{1}+\frac{1}{-\log t} \hat{X}_{2}+o\left((-\log t)^{-1}\right),
\end{aligned}
$$

with $\hat{\phi}_{i j}, \hat{X}_{j}=O(1)$ as $t \rightarrow 0$.

On substituting these expansions into (4.13) and (4.14) we find that, although we obtain a fully nonlinear free boundary problem at leading order, there is a simple, linear solution that reproduces the matching conditions up to a constant. A simple linear solution is also avaliable at $O(\log (-\log t) /(-\log t))$, and we find that

$$
\begin{aligned}
& \hat{\phi}_{i 0}=-\frac{1}{\pi\left(1+\frac{1}{2} \bar{\rho}\right)}\left(-2 \hat{x}+K_{i}\right), \quad \hat{X}_{0}=\frac{1}{\pi\left(1+\frac{1}{2} \bar{\rho}\right)}, \quad(1+\bar{\rho}) K_{1}-K_{2}=\frac{4 \bar{\rho}}{3 \pi\left(1+\frac{1}{2} \bar{\rho}\right)}, \\
& \hat{\phi}_{i 1}=-\frac{1}{\pi\left(1+\frac{1}{2} \bar{\rho}\right)}\left(\hat{x}+L_{i}\right), \quad \hat{X}_{1}=-\frac{1}{2 \pi\left(1+\frac{1}{2} \bar{\rho}\right)}, \quad(1+\bar{\rho}) L_{1}-L_{2}=-\frac{4 \bar{\rho}}{3 \pi\left(1+\frac{1}{2} \bar{\rho}\right)} .
\end{aligned}
$$

The constants $K_{i}$ and $L_{i}$ are not fully determined at this order.

At $O\left((-\log t)^{-1}\right)$ we obtain a nontrivial boundary value problem set in the quarter planes $\hat{y}>0$ and $\hat{x}<\hat{X}_{0}$ for $\hat{\phi}_{12}$ and $\hat{x}>\hat{X}_{0}$ for $\hat{\phi}_{22}$. After defining $\tilde{\phi}_{i} \equiv \hat{\phi}_{i 2}, \tilde{X} \equiv \hat{X}_{2}$, $\tilde{x}=\hat{x}-\hat{X}_{0}$ and $\tilde{y}=\hat{y}$, we arrive at

$$
\begin{gathered}
\tilde{\nabla}^{2} \tilde{\phi}_{i}=0 \text { in } \tilde{\mathcal{D}}_{i}, \\
2 \tilde{X}-2 \tilde{y} \frac{d \tilde{X}}{d \tilde{y}}=\frac{\partial \tilde{\phi}_{i}}{\partial \tilde{x}}+\frac{1}{\pi\left(1+\frac{1}{2} \bar{\rho}\right)} \text { at } \tilde{x}=0, \tilde{y}>0, \\
3\left\{(1+\bar{\rho}) \tilde{\phi}_{1}-\tilde{\phi}_{2}\right\}-2 \tilde{y} \frac{\partial}{\partial \tilde{y}}\left\{(1+\bar{\rho}) \tilde{\phi}_{1}-\tilde{\phi}_{2}\right\} \\
=-\tilde{y}-\frac{2 \bar{\rho}}{\pi\left(1+\frac{1}{2} \bar{\rho}\right)} \tilde{X}-\frac{2 \bar{\rho}}{3 \pi^{2}\left(1+\frac{1}{2} \bar{\rho}\right)^{2}} \text { at } \tilde{x}=0, \tilde{y}>0, \\
\frac{\partial \tilde{\phi}_{i}}{\partial \tilde{y}}=0 \text { at } \tilde{y}=0, \\
\tilde{\phi}_{1} \sim-\frac{1}{\pi\left(1+\frac{1}{2} \bar{\rho}\right)}[\tilde{x}\{\log \tilde{r}+k(\bar{\rho})+\log \operatorname{Ri}\}+\tilde{y}(\pi-\tilde{\theta})] \quad \text { as } \tilde{r} \rightarrow \infty,
\end{gathered}
$$


18

\section{J. Billingham}

$$
\begin{gathered}
\tilde{\phi}_{2} \sim-\frac{1}{\pi\left(1+\frac{1}{2} \bar{\rho}\right)}[\tilde{x}\{\log \tilde{r}+k(\bar{\rho})+\log \mathrm{Ri}\}-\tilde{y} \tilde{\theta}] \quad \text { as } \tilde{r} \rightarrow \infty, \\
\tilde{X} \sim-\frac{1}{2 \pi\left(1+\frac{1}{2} \bar{\rho}\right)}\{\log \tilde{y}+k(\bar{\rho})+1+\log \mathrm{Ri}\} \quad \text { as } \tilde{y} \rightarrow \infty .
\end{gathered}
$$

Note that, at this order in $(-\log t)^{-1}$, the inner region does not see the leading order flow, $\phi_{0}(x, y)$, in these matching conditions, since $\phi_{0}$ is quadratic in $r$ as $r \rightarrow 0$ close to the stagnation point. This is why this inner problem is equivalent to that for a two fluid dam-break problem, with initially stationary fluids.

A key feature of this boundary value problem is the term involving $\bar{\rho} \tilde{X}$ on the right hand side of (4.19). This is the reason that the limit $\bar{\rho} \rightarrow 0$ is singular. It is this term, which arises from the inertial terms in the Bernoulli equation in this accelerating inner region, that allows a bounded solution to exist. Simply differentiating (4.19) with respect to $\tilde{y}$, taking the limit $\tilde{y} \rightarrow 0$ and using 4.20 shows that

$$
\frac{\partial \tilde{X}}{\partial \tilde{y}}(0)=-\frac{\pi\left(1+\frac{1}{2} \bar{\rho}\right)}{2 \bar{\rho}} .
$$

In terms of the original variables, this is

$$
\frac{\partial X}{\partial y}(0) \sim-\frac{\pi\left(1+\frac{1}{2} \bar{\rho}\right)}{2 \bar{\rho}(-\log t)} \text { as } t \rightarrow 0,
$$

which reproduces the result of King \& Needham (1994) and Uddin \& Needham (2015) in the single fluid limit, $\bar{\rho} \rightarrow \infty$. Note that it is also possible to recover equations (3.28) to (3.31) with $\sigma=1$ in Uddin \& Needham (2015) in this limit after a rescaling. In the Boussinesq limit, $\bar{\rho} \rightarrow 0$ with $\mathrm{Ri}=O(1)$, the slope at the contact point and, as we shall see, its position, tend to infinity, and hence this limit is a singular perturbation.

\subsection{Solution of (4.17) to (4.21) using Mellin transforms}

We begin by making two transformations of the dependent variables, $\tilde{\phi}_{1}, \tilde{\phi}_{2}$ and $\tilde{X}$. Firstly, we subtract off the solution at infinity, and rewrite 4.17 to 4.21 in terms of

$$
\begin{gathered}
\bar{\phi}_{1}=\tilde{\phi}_{1}+\frac{1}{\pi\left(1+\frac{1}{2} \bar{\rho}\right)}[\tilde{x}\{\log \tilde{r}+k(\bar{\rho})+\log \operatorname{Ri}\}+\tilde{y}(\pi-\tilde{\theta})], \\
\bar{\phi}_{2}=\tilde{\phi}_{2}+\frac{1}{\pi\left(1+\frac{1}{2} \bar{\rho}\right)}[\tilde{x}\{\log \tilde{r}+k(\bar{\rho})+\log \operatorname{Ri}\}-\tilde{y} \tilde{\theta}], \\
\bar{X}=\tilde{X}+\frac{1}{2 \pi\left(1+\frac{1}{2} \bar{\rho}\right)}\{\log \tilde{y}+k(\bar{\rho})+1+\log \mathrm{Ri}\} .
\end{gathered}
$$

In terms of these variables, the Bernoulli equation, 4.19), becomes

$$
\begin{gathered}
3\left\{(1+\bar{\rho}) \bar{\phi}_{1}-\bar{\phi}_{2}\right\}-2 \tilde{y} \frac{\partial}{\partial \tilde{y}}\left\{(1+\bar{\rho}) \bar{\phi}_{1}-\bar{\phi}_{2}\right\} \\
=-\frac{2 \bar{\rho}}{\pi\left(1+\frac{1}{2} \bar{\rho}\right)} \bar{X}+\frac{\bar{\rho}}{\pi^{2}\left(1+\frac{1}{2} \bar{\rho}\right)^{2}}\left(\log \tilde{r}+k(\bar{\rho})+\frac{1}{3}+\log \mathrm{Ri}\right) \text { at } \tilde{x}=0, \tilde{y}>0 .
\end{gathered}
$$

This indicates that $\bar{\phi}_{1}$ and $\bar{\phi}_{2}$ are of $O(\log \tilde{r})$ as $r \rightarrow \infty$, and suggests a final transformation,

$$
\phi_{i}^{*}=\bar{\phi}_{i}-C_{i} \log \left(1+\tilde{r}^{2}\right)-D_{i}, \quad X^{*}=\bar{X}
$$


The constants $C_{i}$ will be determined below, whilst the constants $D_{i}$ are not determined at this order of matching, but they must satisfy

$$
(1+\bar{\rho}) C_{1}-C_{2}=\frac{\bar{\rho}}{3 \pi^{2}\left(1+\frac{1}{2} \bar{\rho}\right)^{2}}, \quad(1+\bar{\rho}) D_{1}-D_{2}=\frac{\bar{\rho}}{3 \pi^{2}\left(1+\frac{1}{2} \bar{\rho}\right)^{2}}(k+1+\log \mathrm{Ri}) .
$$

This eliminates logarithmic behaviour in the far field without introducing a new singularity at $\tilde{r}=0$. In addition, the symmetry of the problem now shows that $\phi_{1}^{*}(\tilde{r}, \tilde{\theta})=$ $-\phi_{2}^{*}(\tilde{r}, \pi-\tilde{\theta})$.

In terms of these starred variables, in polar coordinates,

$$
\begin{gathered}
\tilde{\nabla}^{2} \phi_{2}^{*}=-\frac{C_{2}}{6 \pi^{2}\left(1+\frac{1}{2} \bar{\rho}\right)^{2}} \tilde{\nabla}^{2} \log \left(1+\tilde{r}^{2}\right) \quad \text { in } \tilde{r}>0,0<\tilde{\theta}<\pi / 2, \\
2 X^{*}-2 \tilde{r} \frac{d X^{*}}{d \tilde{r}}=-\frac{1}{\tilde{r}} \frac{\partial \phi_{2}^{*}}{\partial \tilde{\theta}} \text { at } \tilde{\theta}=\frac{\pi}{2}, \tilde{r}>0, \\
3 \phi_{2}^{*}-2 \tilde{r} \frac{\partial \phi_{2}^{*}}{\partial \tilde{r}}=\frac{\bar{\rho}}{\pi\left(1+\frac{1}{2} \bar{\rho}\right)^{2}} X^{*} \\
+\frac{\bar{\rho}}{6 \pi^{2}\left(1+\frac{1}{2} \bar{\rho}\right)^{3}}\left\{\frac{3}{2} \log \left(\frac{\tilde{r}^{2}}{1+\tilde{r}^{2}}\right)-\frac{2}{1+\tilde{r}^{2}}\right\} \text { at } \tilde{\theta}=\frac{\pi}{2}, \tilde{r}>0, \\
\frac{\partial \phi_{2}^{*}}{\partial \tilde{\theta}}=0 \text { at } \tilde{\theta}=0 .
\end{gathered}
$$

After these transformations, we expect that $\phi_{2}^{*}=O\left(\tilde{r}^{-1}\right)$ and $X^{*}=O\left(\tilde{r}^{-2}\right)$ as $\tilde{r} \rightarrow \infty$ and $\phi_{2}^{*}=o(1)$ and $X^{*}=O(\log \tilde{r})$ as $\tilde{r} \rightarrow 0$. The quarter-plane boundary value problem given by 4.26) to 4.29 is equivalent to that given by (A15) to (A17) in King \& Needham (1994, Appendix A). We refer the reader to this paper for details of the solution using Mellin transforms and the analysis of the solution, $\tilde{X}$, which is given by

$$
\begin{gathered}
\tilde{X}=-\frac{1}{2 \pi\left(1+\frac{1}{2} \bar{\rho}\right)}\{\log \tilde{y}+k(\bar{\rho})+1+\log \mathrm{Ri}\} \\
-\frac{\pi^{1 / 2}}{8\left(1+\frac{1}{2} \bar{\rho}\right)} \frac{1}{2 \pi i} \int_{c-i \infty}^{c+i \infty}\left(\frac{4 \pi\left(1+\frac{1}{2} \bar{\rho}\right)^{2} \tilde{y}}{\bar{\rho}}\right)^{-p} \frac{1}{p(p+1) \Gamma\left(p+\frac{3}{2}\right) \sin \left(\frac{\pi p}{2}\right)} d p
\end{gathered}
$$

with $0<c<2$. By examining the solution for $\phi_{2}^{*}$, we find that in order to remove a logarithmic singularity at $r=0$, we must take

$$
C_{2}=-\frac{\bar{\rho}}{6 \pi^{2}\left(1+\frac{1}{2} \bar{\rho}\right)^{3}} .
$$

The inversion contour can then be shifted to the left and the artificial function $\log \left(1+\tilde{r}^{2}\right)$ in 4.25 removed to give

$$
\begin{gathered}
\tilde{\phi}_{1}=-\frac{1}{\pi\left(1+\frac{1}{2} \bar{\rho}\right)}[\tilde{x}\{\log \tilde{r}+k(\bar{\rho})+\log \mathrm{Ri}\}+\tilde{y}(\pi-\tilde{\theta})] \\
+\frac{\bar{\rho}}{8 \pi^{1 / 2}\left(1+\frac{1}{2} \bar{\rho}\right)^{3}} \frac{1}{2 \pi i} \int_{d-i \infty}^{d+i \infty}\left(\frac{4 \pi\left(1+\frac{1}{2} \bar{\rho}\right)^{2} \tilde{r}}{\bar{\rho}}\right)^{-p} \frac{\cos (p \tilde{\theta})}{p(p+1) \Gamma\left(p+\frac{5}{2}\right) \sin (\pi p)} d p+\text { constant }
\end{gathered}
$$




\section{J. Billingham}

$$
\begin{gathered}
\tilde{\phi}_{2}=-\frac{1}{\pi\left(1+\frac{1}{2} \bar{\rho}\right)}[\tilde{x}\{\log \tilde{r}+k(\bar{\rho})+\log \mathrm{Ri}\}-\tilde{y} \tilde{\theta}] \\
-\frac{\bar{\rho}}{8 \pi^{1 / 2}\left(1+\frac{1}{2} \bar{\rho}\right)^{3}} \frac{1}{2 \pi i} \int_{d-i \infty}^{d+i \infty}\left(\frac{4 \pi\left(1+\frac{1}{2} \bar{\rho}\right)^{2} \tilde{r}}{\bar{\rho}}\right)^{-p} \frac{\cos \{p(\pi-\tilde{\theta})\}}{p(p+1) \Gamma\left(p+\frac{5}{2}\right) \sin (\pi p)} d p+\text { constant }
\end{gathered}
$$

and $-1<d<0$.

Note that if we define

$$
Y=4 \pi\left(1+\frac{1}{2} \bar{\rho}\right)^{2} \tilde{y} / \bar{\rho}, \quad \xi=\left(1+\frac{1}{2} \bar{\rho}\right) \tilde{X}-\frac{1}{2 \pi}\left\{\log \left(\frac{4 \pi\left(1+\frac{1}{2} \bar{\rho}\right)^{2}}{\bar{\rho} \mathrm{Ri}}\right)-k(\bar{\rho})-1\right\},
$$

4.30 can be written as

$$
\xi=-\frac{1}{2 \pi} \log Y-\frac{\pi^{1 / 2}}{8} \frac{1}{2 \pi i} \int_{c-i \infty}^{c+i \infty} Y^{-p} \frac{1}{p(p+1) \Gamma\left(p+\frac{3}{2}\right) \sin \left(\frac{\pi p}{2}\right)} d p,
$$

which contains no parameters. On this contour, if we write $p=c+i s$, the integrand is of $O\left(s^{-(c+4)}\right)$ as $s \rightarrow \infty$. This means that we can shift the contour to the left (see King \& Needham (1994) for a more detailed discussion and justification), picking up contributions from the poles at $p=0,-1$ and -2 , and write

$\xi=\frac{1}{2 \pi}\left(\frac{2}{\pi^{1 / 2}} \Gamma^{\prime}\left(\frac{3}{2}\right)+1\right)-\frac{1}{8} Y-\frac{1}{16 \pi} Y^{2}-\frac{\pi^{1 / 2}}{8} \frac{1}{2 \pi i} \int_{c^{\prime}-i \infty}^{c^{\prime}+i \infty} Y^{-p} \frac{1}{p(p+1) \Gamma\left(p+\frac{3}{2}\right) \sin \left(\frac{\pi p}{2}\right)} d p$,

with $-3<c^{\prime}<-2$. We cannot, however, shift the contour beyond the pole at $p=-3$ since the integral is not convergent for $c^{\prime}<-3$. This suggests that as $Y \rightarrow 0$, there is a term of $O\left(Y^{k}\right)$ with $2<k<3$ in the asymptotic expansion for $\xi$.

By noting that the Mellin transform of $Y^{5 / 2} \sin \left(\frac{1}{Y}+\frac{\pi}{4}\right)$ is

$$
\frac{1}{\left(p+\frac{5}{2}\right)\left(p+\frac{3}{2}\right) \Gamma\left(p+\frac{3}{2}\right) \sin \left(\frac{\pi p}{2}\right)} \times-\frac{\pi \sin ^{2}\left(\frac{\pi p}{2}\right)}{2 \sin ^{2}(\pi p)-1},
$$

for $-\frac{7}{2}<\Re(p)<-\frac{5}{2}$, we can see that this has the same asymptotic behaviour as the integrand in (4.34) as $s \rightarrow \infty$, so we can write

$$
\begin{gathered}
\xi=\frac{1}{2 \pi}\left(\frac{2}{\pi^{1 / 2}} \Gamma^{\prime}\left(\frac{3}{2}\right)+1\right)-\frac{1}{8} Y-\frac{1}{16 \pi} Y^{2}+\frac{1}{4 \pi^{1 / 2}} Y^{5 / 2} \sin \left(\frac{1}{Y}+\frac{\pi}{4}\right) \\
-\frac{\pi^{1 / 2}}{8} \frac{1}{2 \pi i} \int_{c^{\prime}-i \infty}^{c^{\prime}+i \infty} \frac{Y^{-p}}{\Gamma\left(p+\frac{3}{2}\right) \sin \left(\frac{\pi p}{2}\right)}\left\{\frac{1}{p(p+1)}-\frac{2 \sin ^{2}\left(\frac{\pi p}{2}\right)}{\left(p+\frac{5}{2}\right)\left(p+\frac{3}{2}\right)\left(2 \sin ^{2}\left(\frac{\pi p}{2}\right)-1\right)}\right\} d p .
\end{gathered}
$$

The integrand in this expression is of $O\left(s^{-(c+5)}\right)$ as $s \rightarrow \infty$, so we can shift the contour to the left beyond the pole at $p=-3$ and conclude that

$\xi=\frac{1}{2 \pi}\left(\frac{2}{\pi^{1 / 2}} \Gamma^{\prime}\left(\frac{3}{2}\right)+1\right)-\frac{1}{8} Y-\frac{1}{16 \pi} Y^{2}+\frac{1}{4 \pi^{1 / 2}} Y^{5 / 2} \sin \left(\frac{1}{Y}+\frac{\pi}{4}\right)+O\left(Y^{3}\right)$ as $Y \rightarrow 0$.

Figure 11 shows $\xi(Y)$, computed using integral in MATLAB. As expected, close to the contact point there are oscillations of increasing frequency and decreasing amplitude as $Y \rightarrow 0$. This is in contrast to Figure 2 in King \& Needham (1994), which is not plotted at sufficient resolution to reveal these oscillations. The equivalent problem was 

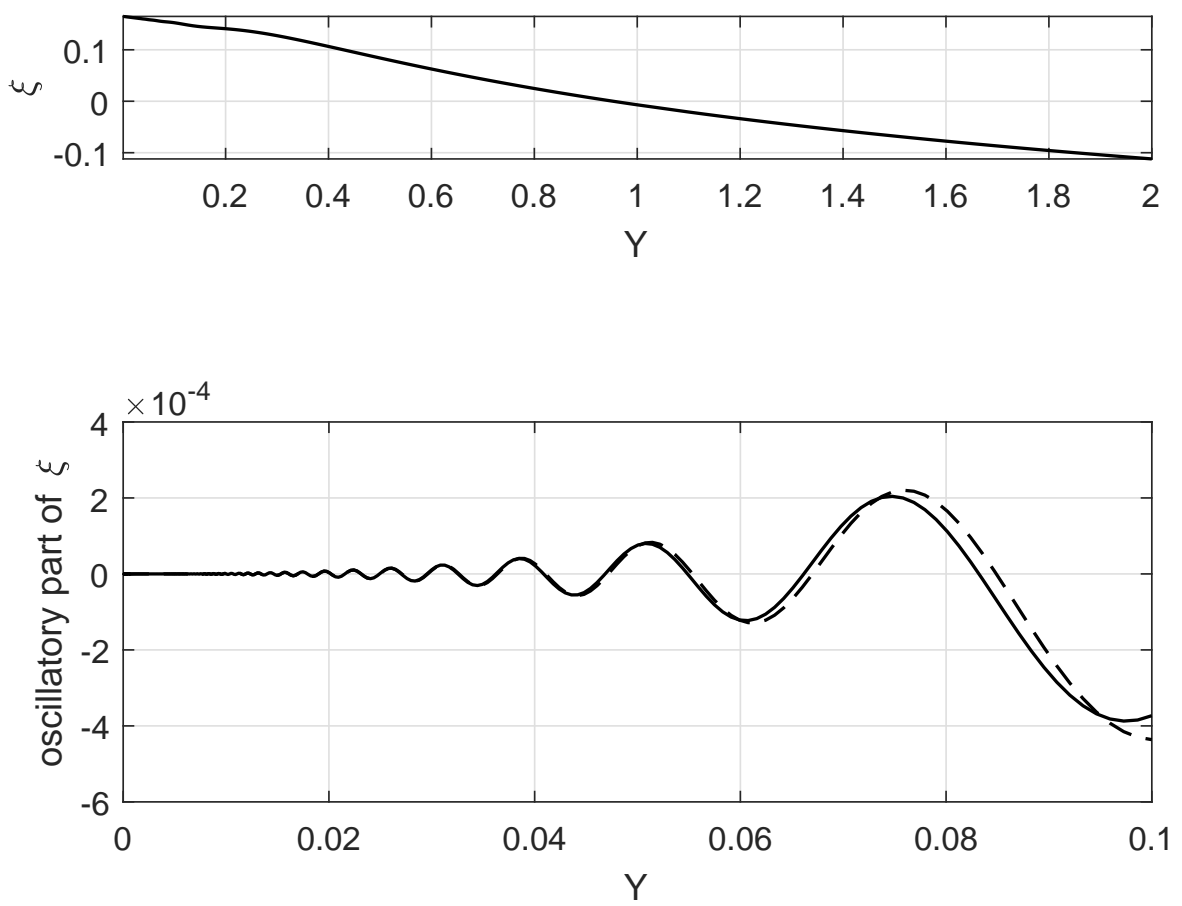

Figure 11. The upper panel shows the function $\xi(Y)$, computed from 4.33). The lower panel shows the oscillatory part, $\xi(Y)-\frac{1}{2 \pi}\left(\frac{2}{\pi^{1 / 2}} \Gamma^{\prime}\left(\frac{3}{2}\right)+1\right)+\frac{1}{8} Y+\frac{1}{16 \pi} Y^{2}$, along with the asymptotic approximation, $\frac{1}{4 \pi^{1 / 2}} Y^{5 / 2} \sin \left(\frac{1}{Y}+\frac{\pi}{4}\right)$, shown as a broken line.

also studied by Korobkin \& Yilmaz (2009) using parameter asymptotics to derive the governing equations and Fourier transforms to solve them, which produced what appears to be the same oscillatory solution (Figure 4). In Needham et al. (2008, Appendix C), similar oscillations were shown to exist close to an inclined accelerating plate. A local analysis of (4.26) to 4.29 above leads to an equation that controls the behaviour of the oscillations which is identical to (C.9) in Needham et al. (2008, Appendix C) with $\gamma=2$, consistent with the asymptotic expansion (4.36). Note that, although $\xi \rightarrow 0$ and $\xi_{Y} \rightarrow 0$ as $Y \rightarrow 0$, the curvature of the free boundary is singular, with $\xi_{Y Y}=O\left(Y^{-3 / 2}\right)$ as $Y \rightarrow 0$. It is straightforward to construct a composite solution, and Figure 12 shows a typical example. Note the huge difference in scales between the main and inset figures, which illustrate the complex structure of the asymptotic solution.

In terms of the original variables, we have

$$
\tilde{X}=\frac{1}{2 \pi\left(1+\frac{1}{2} \bar{\rho}\right)}\left\{\frac{2}{\pi^{1 / 2}} \Gamma^{\prime}\left(\frac{3}{2}\right)-k(\bar{\rho})+\log \left(\frac{4 \pi\left(1+\frac{1}{2} \bar{\rho}\right)^{2}}{\bar{\rho} \mathrm{Ri}}\right)\right\}-\frac{\pi\left(1+\frac{1}{2} \bar{\rho}\right)}{2 \bar{\rho}} \tilde{y}
$$




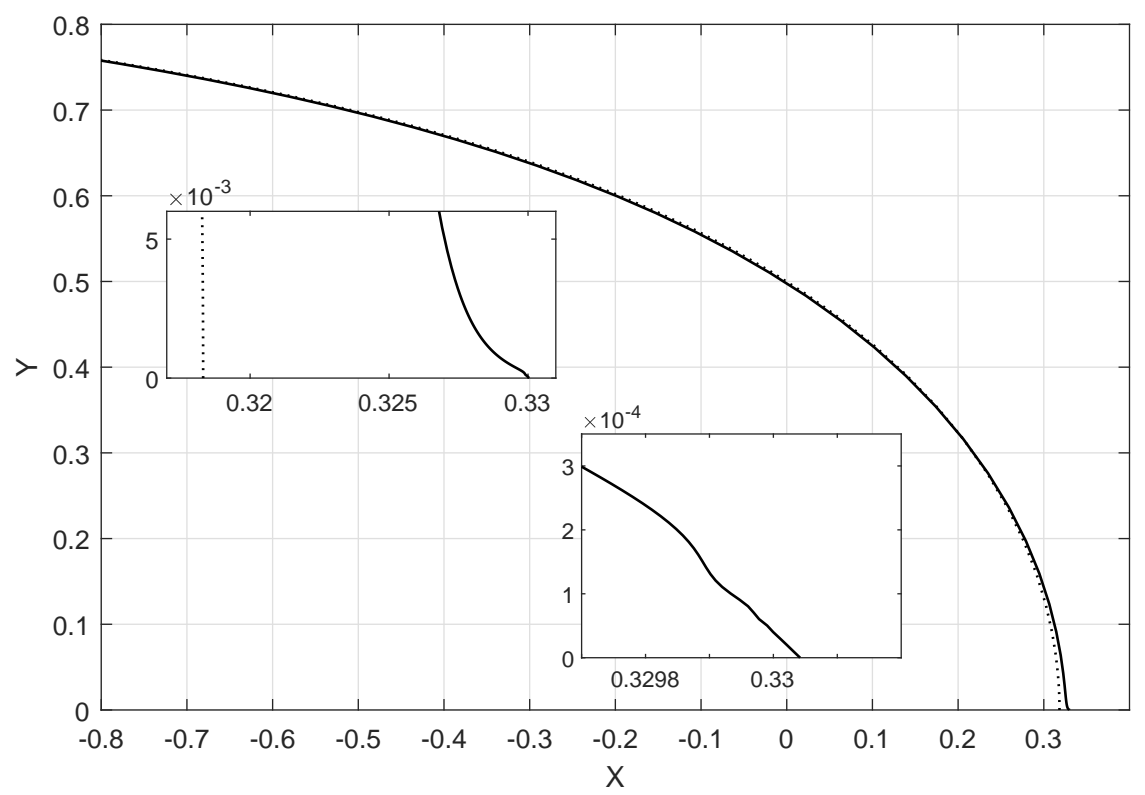

Figure 12. The composite asymptotic solution for $t \ll 1$, with $\bar{\rho}=\mathrm{Ri}=1$ and $t=0.1$. The dotted line shows the initial position of the free boundary, which meets the solid boundary at $x=1 / \pi \approx 0.318$. The inset panels show close ups of the inner region, close to the contact point.

$$
-\frac{\pi\left(1+\frac{1}{2} \bar{\rho}\right)^{3}}{\bar{\rho}^{2}} \tilde{y}^{2}+\frac{16 \pi^{2}\left(1+\frac{1}{2} \bar{\rho}\right)^{4}}{\bar{\rho}^{5 / 2}} \tilde{y}^{5 / 2} \sin \left(\frac{\bar{\rho}}{4 \pi\left(1+\frac{1}{2} \bar{\rho}\right)^{2} \tilde{y}}+\frac{\pi}{4}\right)+O\left(\tilde{y}^{3}\right) \text { as } \tilde{y} \rightarrow 0
$$

consistent with 4.22 . Finally, in terms of the outer variables, the position of the contact point is

$$
X_{c}(t) \sim \frac{1}{\pi}+\frac{\operatorname{Ri} t^{2}}{2 \pi\left(1+\frac{1}{2} \bar{\rho}\right)}\left\{\log \left(\frac{4 \pi\left(1+\frac{1}{2} \bar{\rho}\right)^{2}}{\bar{\rho} \operatorname{Ri} t^{2}(-\log t)}\right)+\frac{2}{\pi^{1 / 2}} \Gamma^{\prime}\left(\frac{3}{2}\right)-k(\bar{\rho})\right\} \quad \text { as } t \rightarrow 0 .
$$

The logarithmic singularity in this expression as $\bar{\rho} \rightarrow 0$ indicates that this is a singular perturbation.

\section{Numerical solution for $\mathbf{R i}=O(1)$}

\subsection{Regularization by a precursor layer}

The natural next step in this investigation is to solve the full, nonlinear initial/boundary value problem numerically. We should, however, pause to take stock of what we now know about the behaviour of the solution. We have discovered that, when $\mathrm{Ri}>0$, the initial flow near the head of the fluid layer is highly spatially oscillatory and develops in a region with lengthscale of $O\left(t^{2}\right)$ as $t \rightarrow 0$, with a singular curvature at the contact point. The intricate, multiscale nature of the free surface close to the boundary when $t \ll 1$ (see Figure 12 means that it is unclear whether it is possible to 
start a simulation using the asymptotic solution as the initial condition. We did attempt to do this, but were unable to develop a numerically stable solution method. In addition, since the asymptotic solution determined in Section 4 is the leading order term in an expansion in powers of $(-\log t)^{-1}$, its accuracy at times small, and yet large enough to use to initialise a numerical simulation, is low.

In the similar, single fluid dam-break flow studied in Uddin \& Needham (2015), the inclusion of weak surface tension regularised the solution at small times. This was also the case in the problem studied in Billingham et al. (2017), where a numerical solution showed that the oscillatory small time solution emerges after a short, surface-tensiondominated transient. Whilst we could do the same here, including surface tension is not really in the spirit of a simplified problem that is meant to be related to powder snow avalanches, for which the small scale behaviour close to the free boundary is better characterised as particle-laden, multiphase turbulence. We therefore decided, in addition to the regularisation provided by the vortex blob method (see discussion below) to modify the initial condition so that a thin layer of fluid 1 (stationary as $x \rightarrow \infty$ in a frame of reference fixed in the solid boundary) lies ahead of the main layer. This is easily achieved by using the streamline given by

$$
X=Y \cot \pi(Y-\epsilon)
$$

as the initial position of the free boundary. In this way, the single fluid solution remains correct when $\bar{\rho}=\mathrm{Ri}=0$, and has $Y \rightarrow \epsilon$ as $X \rightarrow \infty$. Figure 13 shows some numerical solutions for the typical case $\mathrm{Ri}=5, \bar{\rho}=2$, calculated for a sequence of successively smaller values of $\epsilon$, in a frame of reference fixed in the boundary, so that the source (shown as a circle) moves to the right at unit speed. The features displayed in these simulations, namely a depression of the free surface near the original position of the source, overturning of the upper surface of the layer, and overturning at the head of the layer, are, as we shall see, typical. Note that the stopping criterion was that the number of marker points required for the resolution of the free surface that we used reached 1500 which, although somewhat arbitrary, is consistent across the five simulations. Figure 13 provides strong evidence that the value $\epsilon=10^{-3}$, which we have used in the simulations shown below, is a reasonable choice, as the structure of the flow appears no longer to be changing as $\epsilon$ decreases past this value. We have not attempted to construct the asymptotic solution in the limit $\epsilon \rightarrow 0$ in this paper.

Since this regularization removes the contact point, we can also consider looking for steady solutions. Now $Y \rightarrow \epsilon$ and $\phi_{i} \sim-x$ as $x \rightarrow \infty$, so we must modify the Bernoulli constant in 2.4 to give

$$
(1+\bar{\rho})\left(\frac{\partial \phi_{1}}{\partial t}+\frac{1}{2}\left|\nabla \phi_{1}\right|^{2}\right)-\left(\frac{\partial \phi_{2}}{\partial t}+\frac{1}{2}\left|\nabla \phi_{2}\right|^{2}\right)=-\operatorname{Ri}(Y-\epsilon)+\frac{1}{2} \bar{\rho} \text { at } \mathbf{x}=\mathbf{X}(s, t) .
$$

We tried to find steady solutions using a numerical method based on the steady version of that presented below in Section 5.3 and continuation from the known solution when $\bar{\rho}=\mathrm{Ri}=0$. We tried continuation in $\bar{\rho}, \epsilon$ and also in an artificial parameter multiplying the source term. We were, however, unable to obtain converged solutions. If we investigate this further by considering the asymptotic solution for $\bar{\rho} \ll 1$, the equivalents of (3.4) and (3.11) show that now

$$
\frac{d \mu}{d \theta}=\left(\frac{\theta+\epsilon \pi}{\sin ^{2} \theta}\right)\left(-\overline{\mathrm{Ri}} \frac{\theta}{\pi}-\frac{\sin ^{2} \theta}{2(\theta+\epsilon \pi)^{2}}+\frac{\sin \theta \cos \theta}{\theta+\epsilon \pi}\right),
$$

which is of $O\left(\theta^{-1}\right)$ as $\theta \rightarrow 0$. This leads to a non-integrable singularity in the the vortex 

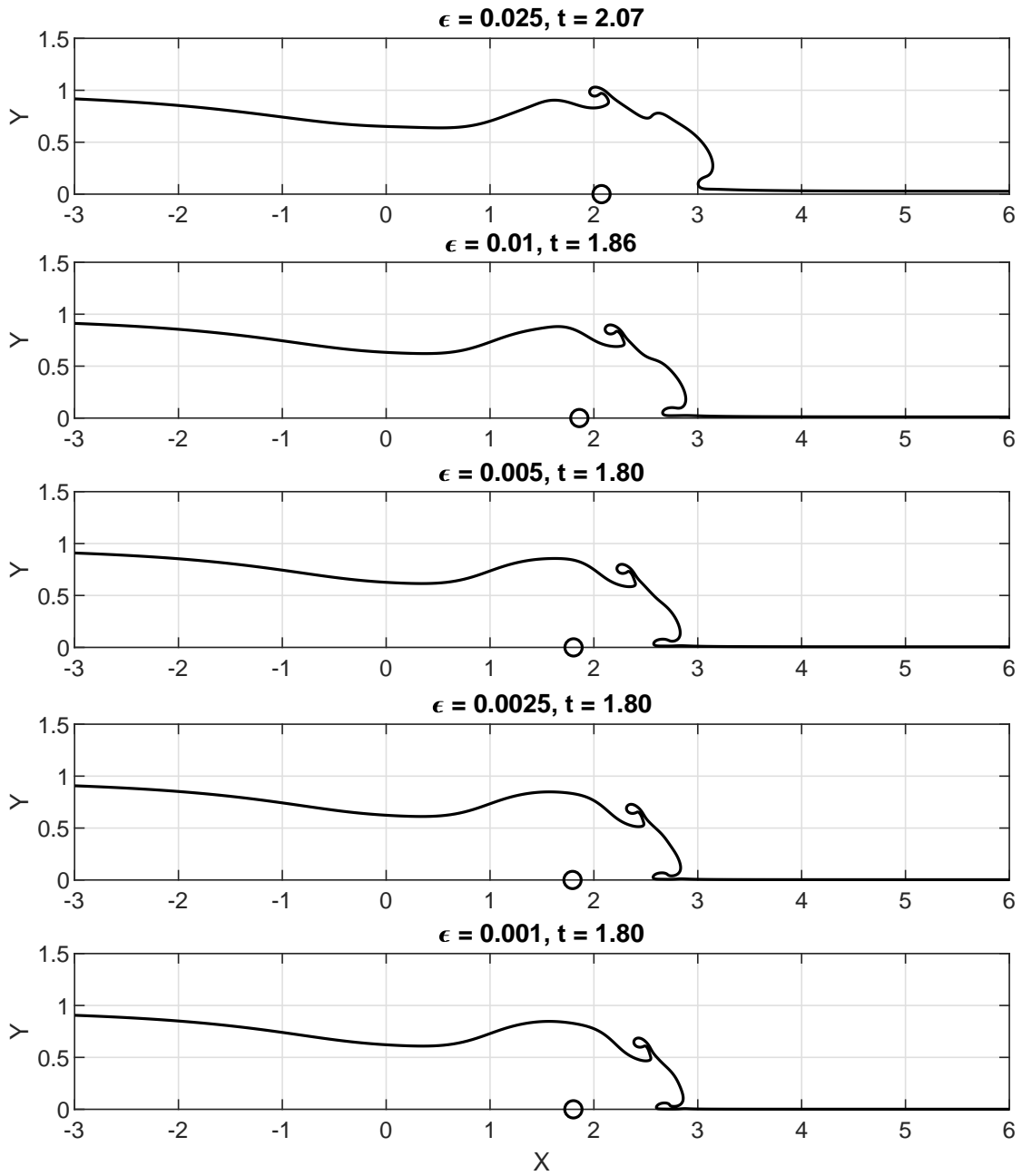

Figure 13. The numerical solution when $\mathrm{Ri}=5, \bar{\rho}=2$ and $\delta=0.05$ for various values of $\epsilon$ at the time when the number of marker points reaches 1500 .

sheet representation, 3.10 , which shows that a finite solution does not exist, consistent with our failed attempts to use numerical continuation starting from $\bar{\rho}=0$. All of this strongly suggests that no steady solution of the regularized problem with $\epsilon>0$ exists, and hence that the steady solution $\phi_{i}=-x+\frac{1}{\pi} \log r$ is structurally unstable to small perturbations in the density of the fluid below a streamline. This does not, however, mean that the unsteady solution that evolves from initial conditions based on this special solution are not interesting and of possible relevance to powder snow avalanches.

\subsection{The adaptive vortex blob method}

It is well-known that periodic, two-fluid, inviscid, irrotational flow with a vortex sheet is ill-posed, Ebin (1988), and that Moore singularities develop in finite time, Moore 
(1978). When surface tension is included, the singularity is avoided, and instead the free surface forms Kelvin-Helmholz rolls. Spectrally-accurate simulations of this roll-up have been performed on geometrically simple problems with periodic initial conditions, regularised either by surface tension (for example, Hou et al. (1994)) or by using a vortex blob method (for example, Baker \& Pham (2006)). We therefore choose to use the latter method, in which the vortex sheet is replaced with a distribution of vorticity.

There are some remarks that we should make that arise from the aperiodic nature of the flow that we are interested in. Firstly, we are not concerned here with the details of the Kelvin-Helmholz roll-up process. We want to understand the bulk dynamics of the flow, and determine where roll-up starts to occur, but appreciate that the details of the roll-up will depend on the regularisation used. Secondly, in previous work using the vortex blob method, the grid spacing in the periodic domain of solution was constant. We will use an adaptive boundary integral method, with a time-dependent, non-uniform grid size that allows us to resolve regions of the boundary where small scale features develop. We must therefore decide how to choose $L_{\delta}$, the lengthscale over which the vorticity is distributed. We will consider two approaches. In Method 1, we take the lengthscale to be the grid size, $L_{\delta}=\Delta s$. This is equivalent to a choice taken by Baker \& Beale (2004), which was shown to lead to accurate results for periodic flow without adaptive regridding. In Method 2, we take $L_{\delta}=\max (\Delta s, \delta)$, with $\delta>0$ a constant. Here, $\delta$ is regarded as a regularisation parameter that gives the lengthscale over which vorticity is smeared, which we can think of as a physical quantity that characterises sub-element scale processes, such as turbulence and particle-particle interactions. Using Method 1 (which we note is equivalent to Method 2 with $\delta=0$ ), we are able to compute the development of a Moore singularity up to large curvatures, but, because we adaptively regrid the boundary to resolve the local curvature, the singularity is not removed, and we cannot compute beyond it.

The effect of varying $\delta$ in the typical example, $\mathrm{Ri}=5, \bar{\rho}=2$, is shown in Figure 14 (note the different scales on the axes). When $\delta=0$, the solution terminates at a developing singularity in the curvature near the head of the layer, as shown in Figure 15. In contrast, using Method 2, the Moore singularity is regularised, so we can integrate beyond its formation time and compute an interface that rolls up and overturns. These rolls start to form at the same time and place as in the solution for $\delta=0$. As $\delta$ increases, the onset of overturning is delayed and the lengthscale of the rolls increases. In the simulations shown below, we have used $\delta=0.05$.

\subsection{Numerical method}

In order to solve the initial/boundary value problem given by $(2.2)$ to $(2.8)$, it is natural to use a boundary integral method. Consider a set of marker points in the boundary, $\partial \mathcal{D}$, parameterised by $\alpha$, that move with velocity $\mathbf{U} \equiv U \mathbf{t}+(\mathbf{u} \cdot \mathbf{n}) \mathbf{n}$, so that

$$
\frac{d \mathbf{X}}{d t}=\mathbf{U}
$$

where $d / d t$ is the Lagrangian time derivative. The normal component of this velocity matches that of the two fluids, but the tangential component is determined, as we shall see below, by specifying the spacing of the surface marker points. We define the unnormalised vortex sheet strength to be $\gamma \equiv \mu_{\alpha}(\alpha, t)$. If $\gamma$ is known, the velocity of the fluid is given, using derivatives of the Plemmelj formula 4.9 , and an image of the free surface in the 

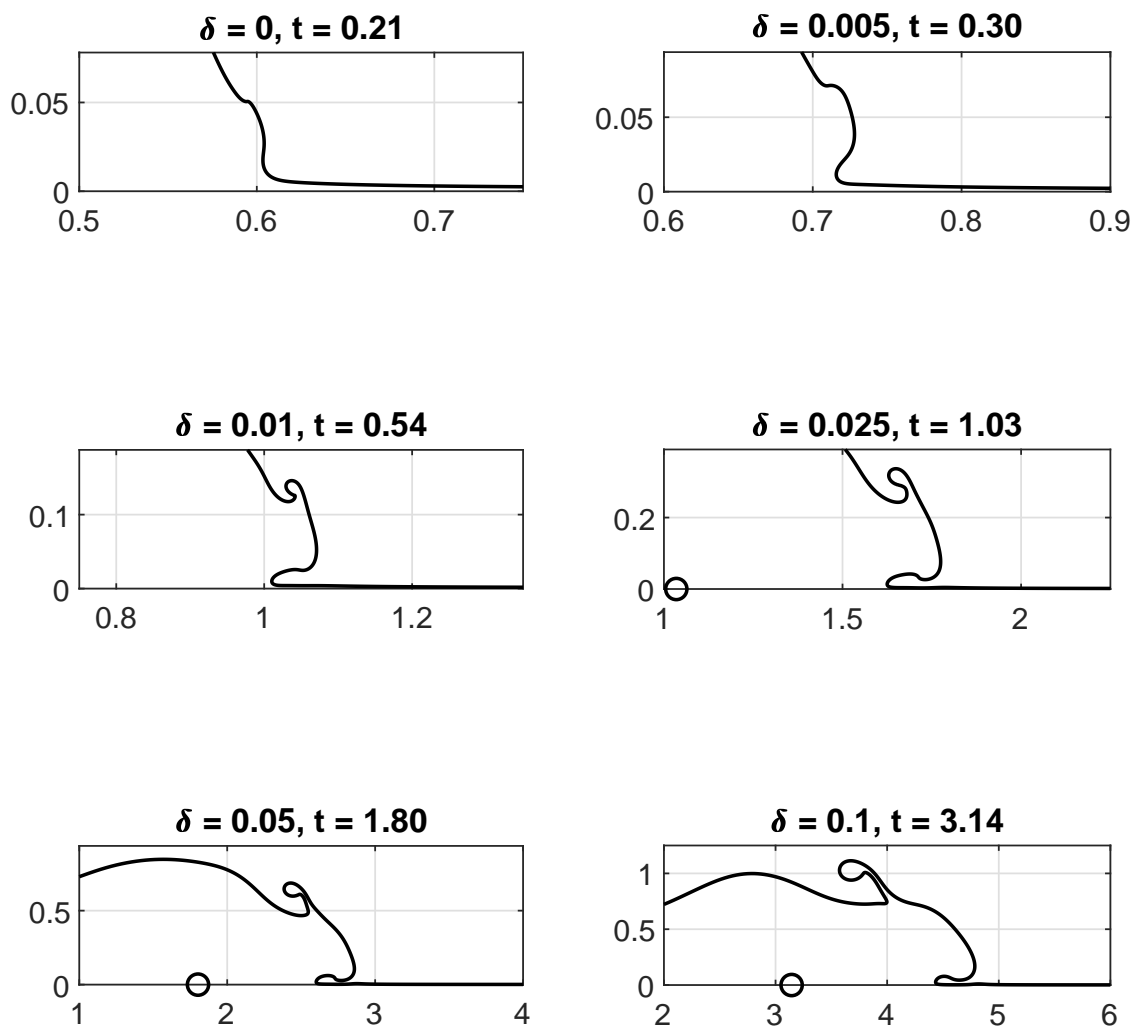

Figure 14. The solution for $\bar{\rho}=2, \mathrm{Ri}=5, \epsilon=10^{-3}$ and various values of $\delta$. The solution is plotted in a frame of reference where the outer fluid is initially stationary at infinity and the source (indicated as a small circle) moves to the right at unit speed. Note the different scales for the axes in each panel. The simulation with $\delta=0$ was terminated when the maximum curvature was greater than 1000 .

solid boundary, by

$$
W=-1+\frac{1}{\pi z}+\frac{1}{2 \pi i} \int_{-\infty}^{\infty} \gamma(\alpha, t)\left\{\frac{1}{Z(\alpha, t)-z}-\frac{1}{Z^{*}(\alpha, t)-z}\right\} d \alpha,
$$

where $z=x+i y, Z(\alpha, t)=X(\alpha, t)+i Y(\alpha, t), W=u-i v$ and $\mathbf{u}=(u, v)$. The first two terms on the right hand side of (5.5) are the unit flow and unit source, which means that $\gamma=0$ when $t=0$. Following Baker et al. (1982) and Hou et al. (1994), the dynamic boundary condition can be manipulated to find the evolution equation for $\gamma$, as

$$
\frac{d \gamma}{d t}-\frac{\partial}{\partial \alpha}\left\{\frac{\gamma}{s_{\alpha}}(U-\mathbf{t} \cdot \mathbf{W})\right\}=
$$




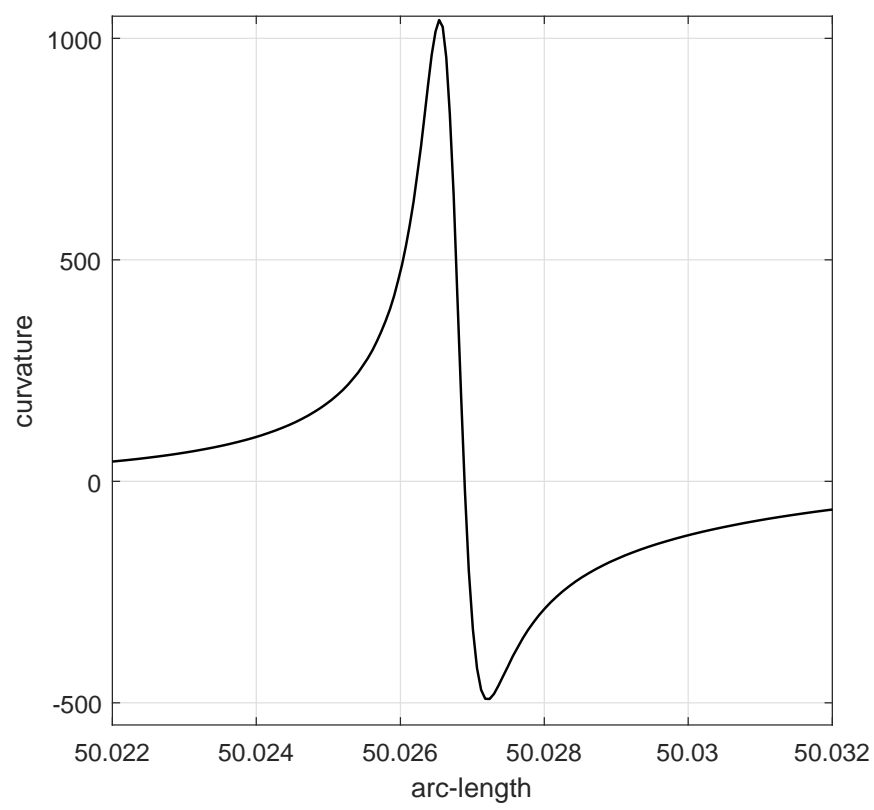

FIGURE 15. The curvature of the free boundary for the solution with $\delta=0$ shown in Figure 14, close to the developing singularity in the curvature.

$$
-\frac{\mathrm{Ri}}{1+\frac{1}{2} \bar{\rho}} Y_{\alpha}-\frac{\bar{\rho}}{1+\frac{1}{2} \bar{\rho}}\left\{s_{\alpha} \mathbf{t} \cdot \frac{d \mathbf{W}}{d t}+\frac{\partial}{\partial \alpha}\left(\frac{\gamma^{2}}{8 s_{\alpha}^{2}}\right)-(U-\mathbf{t} . \mathbf{W}) \mathbf{t} \cdot \mathbf{W}_{\alpha}\right\},
$$

where $s(\alpha, t)$ is arclength and $\mathbf{W}$ is the fluid velocity at the interface given by the principal value integral (5.5). Note that $\mathbf{n} \cdot \mathbf{W}$ is continuous at the boundary, but $\mathbf{t} \cdot \mathbf{W}$ is not, and jumps by an amount $\gamma / s_{\alpha}$ across the boundary. Note also that, since the first step in the lengthy derivation of $(5.6)$ is the differentiation of $\sqrt{5.2})$ with respect to $\alpha$, the Bernoulli constant does not appear. The Bernoulli constant in (5.2) simply reflects the upstream boundary conditions, which are satisfied by the initial conditions in the evolution problem that we solve here. In all the solutions shown below, $\gamma \rightarrow 0$ as $\alpha \rightarrow \pm \infty$, as we would expect since the far field is in equilibrium both up- and downstream.

As an aside, this form of the dynamic boundary condition makes it very straightforward to analyse the motion of the contact point, $x=X_{c}(t)$, (which we have avoided in this formulation by taking $\epsilon>0$ ) where continuity of both components of the fluid velocity means that $U=\mathbf{t} \cdot \mathbf{W}$ and $\gamma=0$. Only two terms in 5.6) are therefore non-zero at the contact point, and we obtain

$$
\frac{d^{2} X_{c}}{d t^{2}}=-\frac{\operatorname{Ri}}{\bar{\rho}} \frac{d Y}{d X}(0) \text { for } \mathrm{Ri}>0,
$$

consistent with the results of Section 4 , but valid for all $t>0$. This relationship between the acceleration of the contact point and the slope of the free boundary at the point of contact shows that, for nonzero Richardson number, the initial acceleration is infinite when $t=0$, with the results of Section 4 giving $d^{2} X_{c} / d t^{2}=O(-\log t)$ as $t \rightarrow 0$. It also shows that any steady solution must have $d Y / d X=0$ at the contact point, i.e. the free surface must be tangent to the boundary. We have been unable to construct a nontrivial local solution of this nature, which suggests that steady solutions are ruled out 
by the behaviour of the solution close to the contact point. In terms of physical variables, abusing our notation for one equation,

$$
\frac{d^{2} X_{c}}{d t^{2}}=-g \frac{d Y}{d X}(0)
$$

Finally, when $\mathrm{Ri}=0$, (5.6) shows that $d X / d Y=0$ at $s=0$, i.e. that the free surface is perpendicular to the boundary at the contact point when gravity is negligible.

Following Baker \& Beale (2004), we define a vortex blob method by modifying (5.5) to be

$W=-1+\frac{1}{\pi z}+\frac{1}{2 \pi i} \int_{-\infty}^{\infty} \gamma(\alpha, t)\left\{\frac{g\left(-|Z(\alpha, t)-z| / L_{\delta}\right)}{Z(\alpha, t)-z}-\frac{g\left(-\left|Z^{*}(\alpha, t)-z\right| / L_{\delta}\right)}{Z^{*}(\alpha, t)-z}\right\} d \alpha$.

We use the lowest order choice of regularisation function, $g(r)=1-e^{-r^{2}}$, as discussed by Baker \& Beale (2004), since we are not as concerned with the details of the flow after the curvature singularity has been regularised, and because we use a low order spatial discretisation. We truncate the surface and discretise using Lagrangian markers and linear elements, with $X=X_{j}, Y=Y_{j}, \gamma=\gamma_{j}$ and $U=U_{j}$ at $\alpha=j$, for $j=1$, $2, \ldots, N$, so that there are $4 N$ unknowns. The integral in 5.9 is evaluated using four point Gaussian quadrature. The parameter $\alpha$ is taken to be gridpoint number, and all spatial derivatives are calculated using central differences. Timestepping is done using the implicit midpoint method, solving the resulting system of nonlinear algebraic equations using quasi-Newton iteration at each timestep. We find that we can reuse the Jacobian, the calculation of which using finite differences is the main numerical bottleneck in the simulation, over multiple timesteps. Note also that the simple, linear representation of the free surface means that only a small portion of the integral in 5.9 has to be recalculated when finding the Jacobian, significantly speeding up the calculation. We chose to use an implicit timestepping method in order to reduce numerical instability and to be able to time step for accuracy rather than stability. In spite of this, for sufficiently large Ri, there is a weak, gridscale instability in $\gamma$, which we damp using five point smoothing at each timestep (c.f. Longuet-Higgins \& Cokelet (1976)). The kinematic and dynamic boundary conditions, (5.4) and (5.9) provide $3 N$ algebraic equations, and we also specify the required element sizes/marker point separations, $\Delta s_{j}$, using

$$
\left(X_{j+1}-X_{j}\right)^{2}+\left(Y_{j+1}-Y_{j}\right)^{2}=\Delta s_{j}^{2} \text { for } j=1,2, \ldots, N-1 .
$$

This allows us to control the tangential spacing of the marker points at each timestep, which is possible because we use implicit timestepping and solve for the tangential marker point velocity, $U$. At each timestep we check whether the curvature of the boundary is adequately resolved, and if not, regrid using quintic splines, so that the curvature of the surface is represented by a cubic spline.

We begin our discussion by making a comparison between the asymptotic solution for $\bar{\rho} \ll 1$, which we constructed in Section 3 and the numerical solution of the fully nonlinear free boundary problem.

\subsection{Comparison of numerical and asymptotic solutions for small $\bar{\rho}$}

We first consider the solution when $\mathrm{Ri}=0$ and $\bar{\rho}=0.01$. Note that when $\mathrm{Ri}=0$, there is no initial singularity at the contact point, so we solved the original problem, with no precursor layer $(\epsilon=0)$. Figure 16 shows the position of the contact point as a function of time. The agreement between the asymptotic and numerical solutions is excellent at relatively small times, but has a later discrepancy, which is within the range 


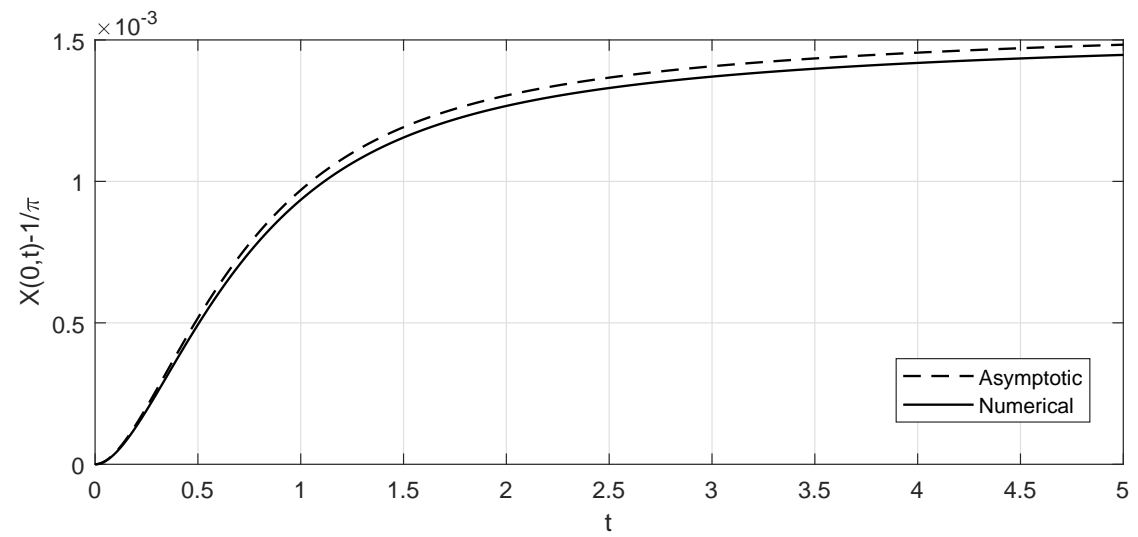

Figure 16. The position of the contact point as a function of time as calculated from the small $\bar{\rho}$ asymptotic and numerical solutions for $\mathrm{Ri}=0$ and $\bar{\rho}=0.01$.
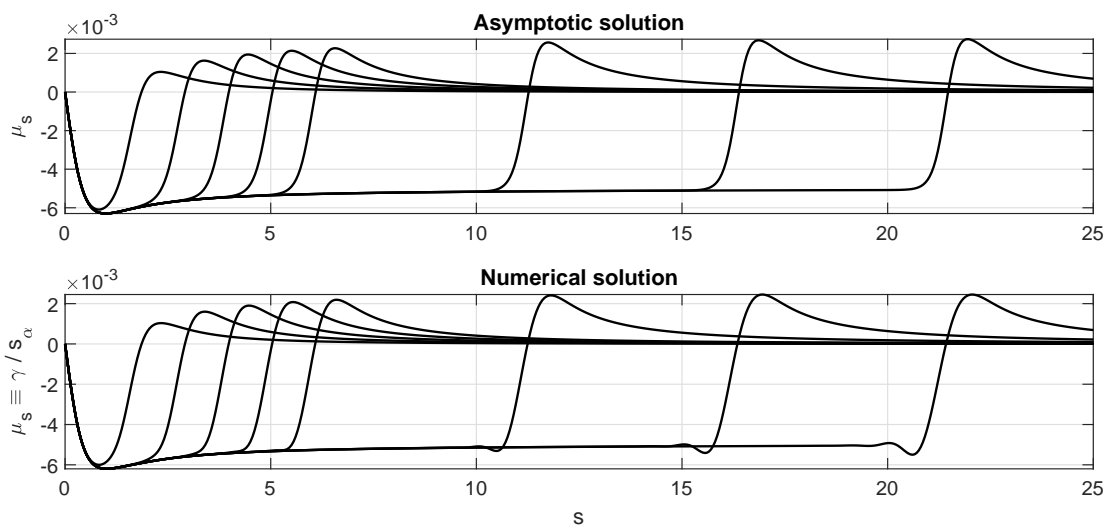

FIGURE 17. The small $\bar{\rho}$ asymptotic (upper panel) and numerical (lower panel) solution for $\mu_{s}$ with $\mathrm{Ri}=0$ and $\bar{\rho}=0.01$ when $t=1,2,3,4,5,10,15$ and 20.

we would expect given the accuracy of the spatial discretisation of the free boundary in the numerical solution. At times before approximately $t=5$, there is good agreement between the numerical and asymptotic solutions in this case, for both the free surface and $\gamma$. This can be most clearly seen in Figure 17, which shows $\mu_{s} \equiv \gamma / s_{\alpha}$ at various times up to $t=20$. The agreement between asymptotic and numerical solutions at earlier times is clear. At later times, $\mu_{s}$ steepens, as expected, but in addition, oscillations develop, which, within the framework of a weakly nonlinear theory, indicates the formation of a dispersive shock. It should be possible to develop a weakly nonlinear theory, but we have not attempted this here. The comparison between numerical and asymptotic solutions for $\mu_{s}$ is a good test of the numerical method, since the asymptotic solution comes from the analytical solution of the simple, semilinear equation (3.4), whilst the numerical solution for $\gamma$ comes from solving the evolution equation (5.6) on the evolving free boundary. Figure 18 shows a comparison of the positions of the free surface at later times, when an increasing discrepancy develops between the asymptotic and numerical solutions. This is due not to discretisation error, but to nonlinearity affecting the deepening depression in the free surface. As the slope of the free surface increases, oscillations form at the rear of the depression, which are coupled to the oscillation in $\mu_{s}$ that we can see in 

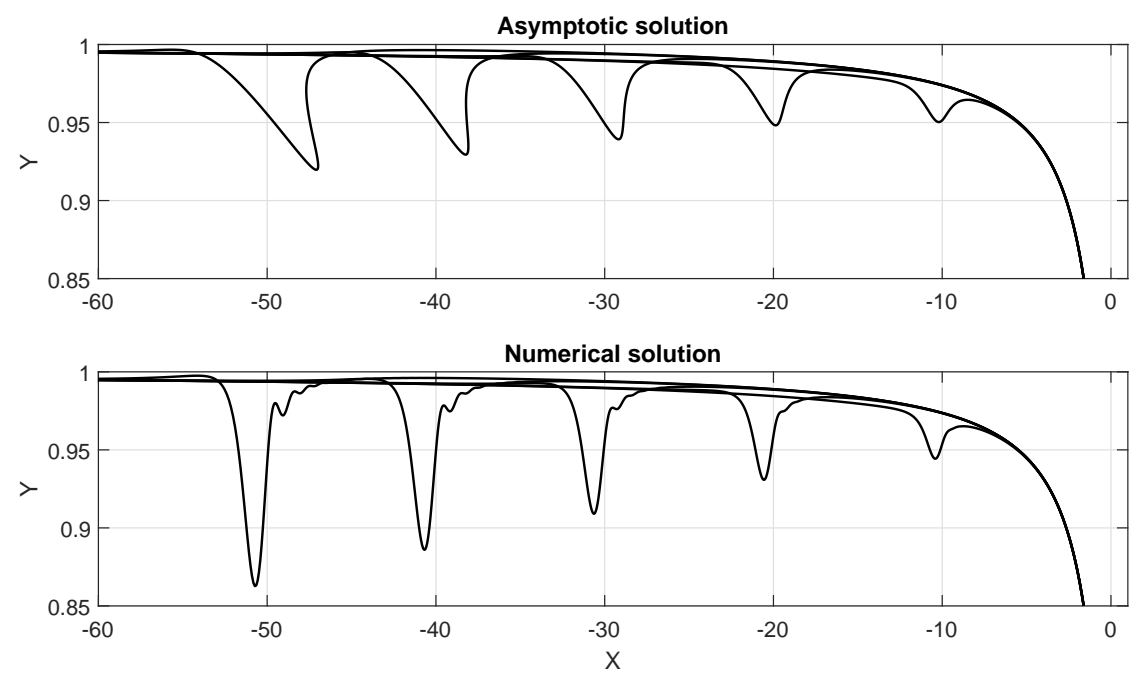

FiguRE 18. The small $\bar{\rho}$ asymptotic (upper panel) and numerical (lower panel) solutions for $\mathrm{Ri}=0$ and $\bar{\rho}=0.01$ when $t=10,20,30,40$ and 50. Note the different scales of the axes.
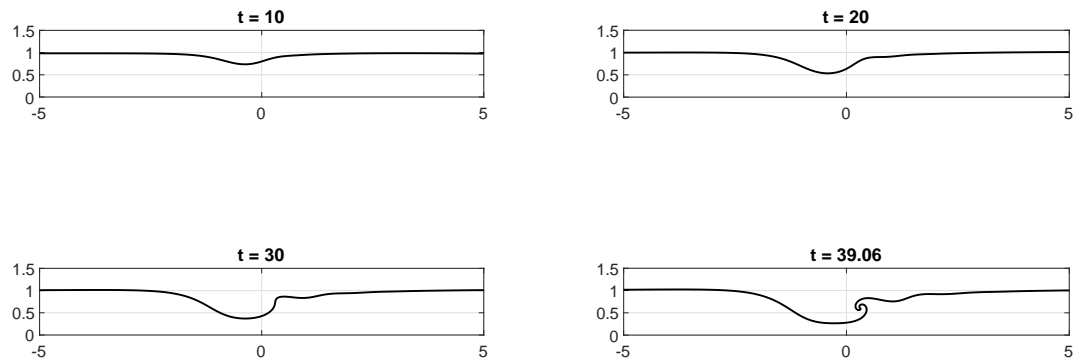

Figure 19. The numerical solution when $\bar{\rho}=0.1, \mathrm{Ri}=0$ and $\delta=0.05$.

Figure 17. The numerical solution shown was computed using Method 1, and we expect that it will terminate in a curvature singularity at some later time, beyond the reach of our numerical method. We can however see this occuring for slightly larger $\bar{\rho}$, so we now consider the numerical solution when $\mathrm{Ri}=0$ and $\bar{\rho}=0.1$. This value is not particularly small, but leads to behaviour qualitatively similar to the solution for $\bar{\rho} \ll 1$, as shown in Figure 19. For this solution, which was computed with $\delta=0.05$, we can also see the depression in the free surface, followed by steepening and the development of a dispersive shock, but now we can solve at times large enough that overturning and the formation of a Kelvin-Helmholz roll can clearly be seen. Solutions for non-zero values of Ri (and $\epsilon=10^{-3}$ ) are shown in Figure 20. As Ri increases, the position of the Kelvin-Helmholz roll moves towards the head of the flow. Note that for small enough $\bar{\rho}$, as is the case in Figure 20, there is no overturning of the surface at the head of the layer. We should not expect the solutions in Figure 20 to agree with the asymptotic solution for $\bar{\rho} \ll 1$, since this was constructed on the basis that $\mathrm{Ri}=O(\bar{\rho})$. 
$R \mathbf{i}=1, t=8.59$

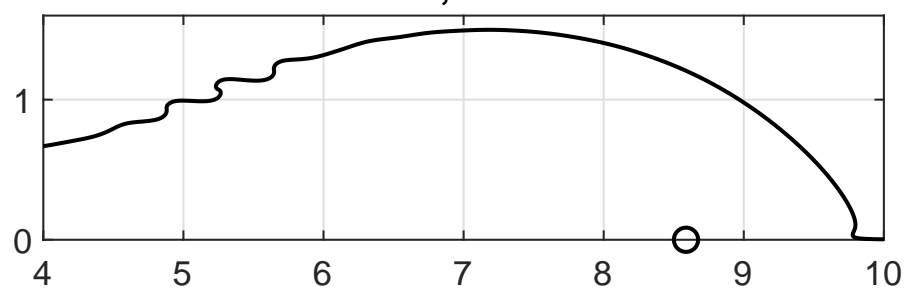

$R \mathbf{i}=2, \mathrm{t}=2.61$

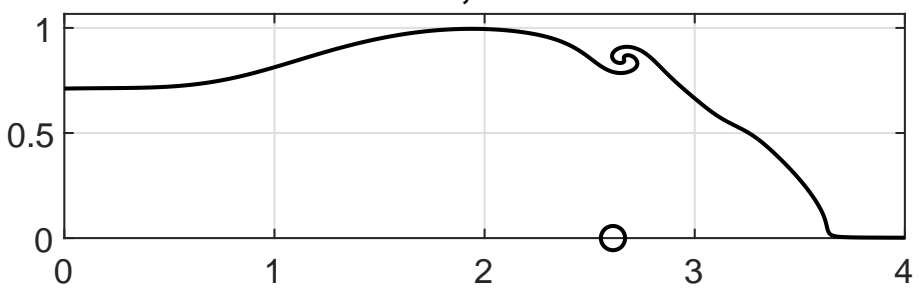

$R \mathbf{i}=5, \mathrm{t}=1.12$

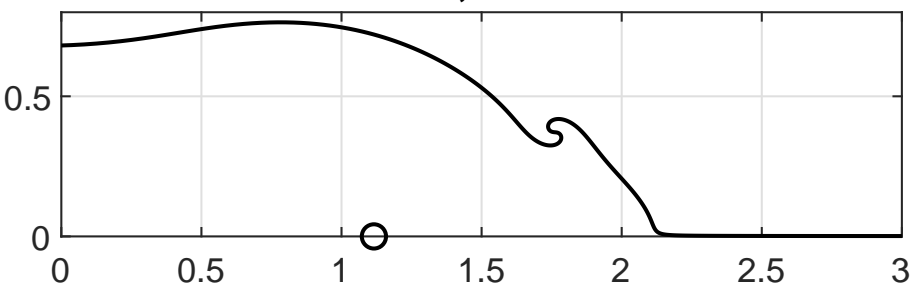

$R \mathbf{i}=10, t=0.81$

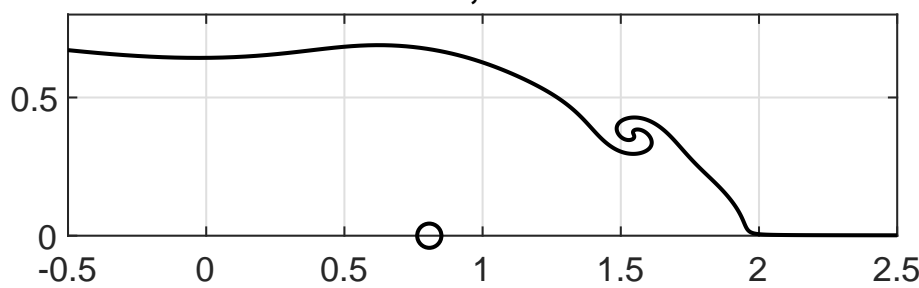

Figure 20. The numerical solution when $\bar{\rho}=0.1$ and $\epsilon=10^{-3}$ for various values of Ri. Note the different scales on the axes.

\subsection{Numerical solutions when $\bar{\rho}$ is not small}

Figures 21 to 23 show the effect of varying $\bar{\rho}$ with $\mathrm{Ri}$ fixed at $0.1,2$ and 10 in successive Figures. Movies that show the evolution of the surface for these, and other, combinations of $\mathrm{Ri}$ and $\bar{\rho}$ can be found at https://drive.google.com/drive/folders/ 1SWVQmdHWTXUCt55kia23xa66Vo_THNlm?usp=sharing. When Ri $=0.1$ (Figure 21), and hence the effect of gravity is weak, the general tendency is for Kelvin-Helmholz instability to occur on the upper surface of the layer, with larger values of $\bar{\rho}$ leading to a stronger Bernoulli effect, and fluid being projected upwards more strongly. There is no sign of any overturning at the head of the layer when $\mathrm{Ri}=0.1$. When $\mathrm{Ri}=2$, and the effect of 


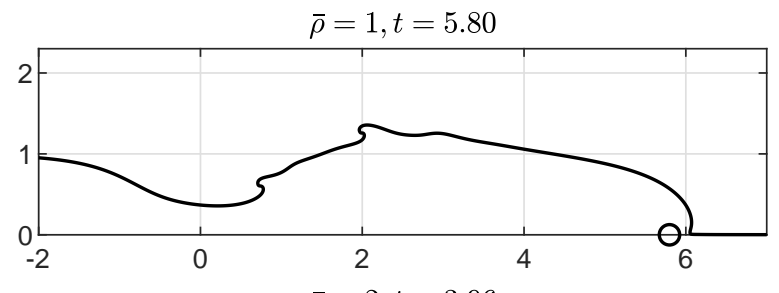

$\bar{\rho}=2, t=3.96$

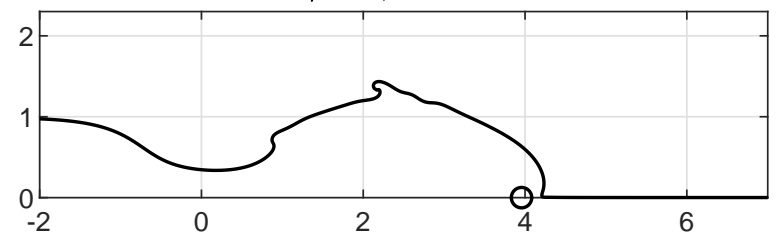

$\bar{\rho}=5, t=3.63$
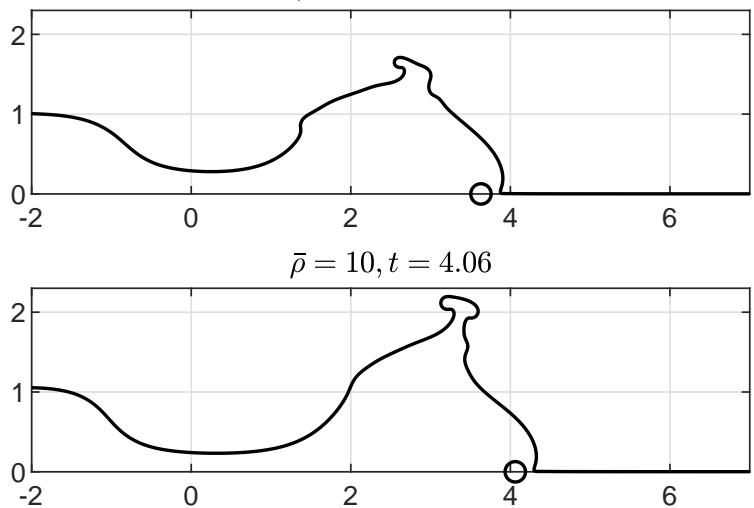

FiguRE 21. Numerical solutions when $\mathrm{Ri}=0.1$ for various values of $\bar{\rho}$.

gravity is stronger (Figure 22) overturning can also occur at the head of the layer, but for sufficiently large $\bar{\rho}$, the initial overturning region is transported along the layer, and fluid is again projected upwards. When $\mathrm{Ri}=10$ (Figure 23), and the effect of gravity is even stronger, overturning at the head of the layer is the dominant effect for sufficiently large $\bar{\rho}$.

\section{Conclusion}

In this paper we have considered the two-dimensional, irrotational flow of a layer of inviscid fluid injected into another inviscid fluid by a source on the boundary moving at constant speed. We were motivated by the work of Caroll et al. (2012), which suggested that this flow provides a very simple model for a powder snow avalanche. With this in mind, after finding that the inital, dam-break dynamics gives rise to complex, multiscale behaviour near the intersection point between the fluid layer and the solid boundary, we chose to regularise this motion by including a thin precursor layer ahead of the original fluid layer. In addition, we chose to deal with the Moore singularities that inevitably arise in an inviscid two fluid flow by using a vortex blob method instead of the more 


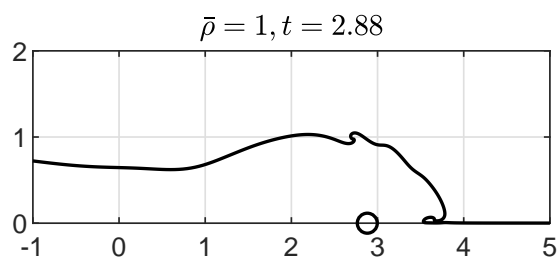

$\bar{\rho}=2, t=2.95$

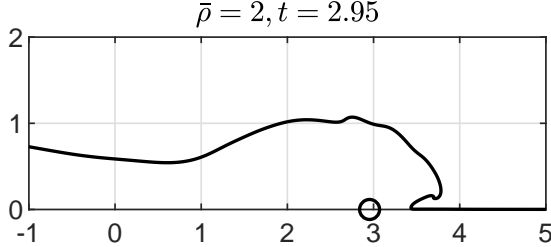

$\bar{\rho}=5, t=3.93$

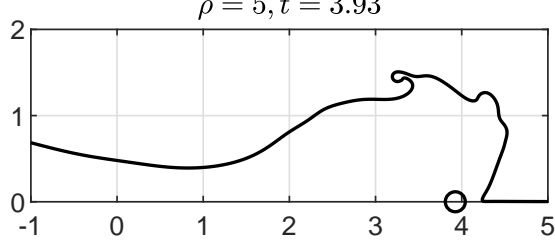

$\bar{\rho}=10, t=4.34$

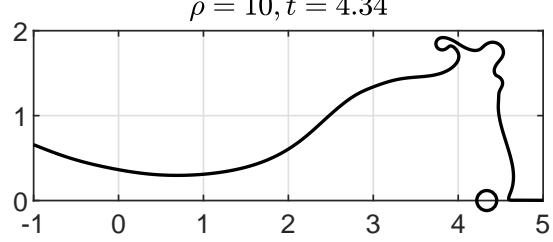

FiguRE 22. Numerical solutions when $\mathrm{Ri}=2$ for various values of $\bar{\rho}$.

usual surface tension regularisation, which is not appropriate in the context of powder snow avalanches. We also introduced an adaptive vortex blob method. This can either be used to integrate up to the formation of a Moore singularity (Method $1, \delta=0$ ), or, by choosing $\delta>0$ to be a dimensionless length that characterises small scale processes not modelled by inviscid, irrotational flow, to compute the roll up of the interface on this lengthscale (Method 2). We found that for small enough dimensionless density difference or Richardson number, the interface rolls up and overturns away from the head of the flow, but that once both the Bernoulli effect and gravity are strong enough, overturning occurs at the head of the layer.

There are some issues that arise from this work, which were mentioned in the paper. Firstly, it would be interesting to derive the weakly nonlinear evolution equation for the free boundary when $\bar{\rho} \ll 1$ and $t=O\left(\bar{\rho}^{-1}\right)$, which numerical solutions suggest should describe the formation of a dispersive shock. Secondly, the structure of the asymptotic solution when $\epsilon \ll 1$, and its relation to the asymptotic solution when $\epsilon=0$ and $t \ll 1$ is not clear, and is a challenging problem to solve using the method of matched asymptotic expansions. Finally, although it is not relevant to avalanche flows, regularisation of the singular solution when $\epsilon=0$ and $t \ll 1$ by the inclusion of surface tension would be of 


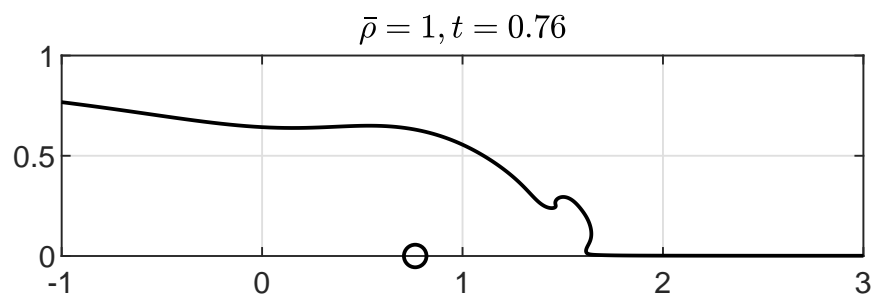

$\bar{\rho}=2, t=0.92$

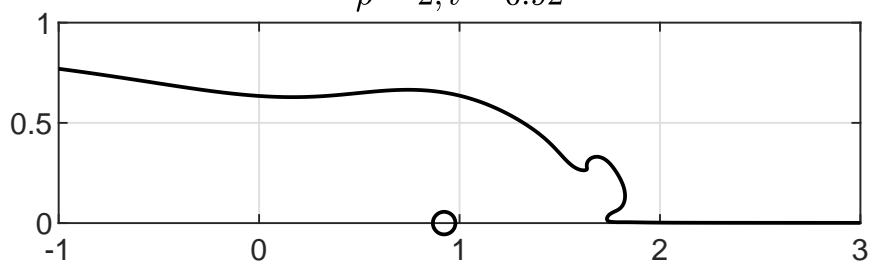

$\bar{\rho}=5, t=1.46$

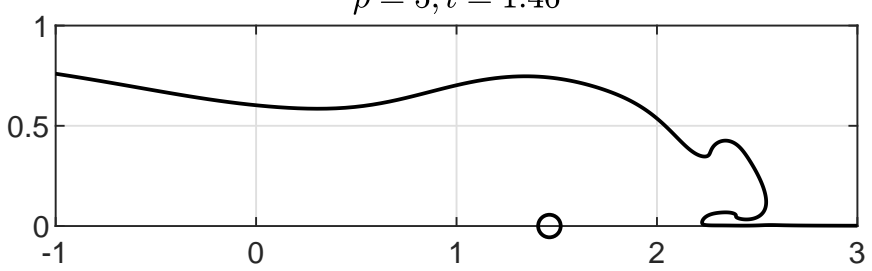

$\bar{\rho}=10, t=1.59$

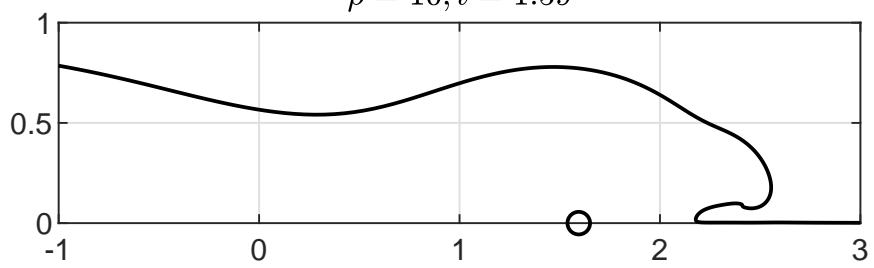

FiguRE 23. Numerical solutions when $\mathrm{Ri}=10$ for various values of $\bar{\rho}$.

interest in other two-fluid dam-break problems (see Uddin \& Needham (2015) for the single fluid dam-break problem).

Other extensions to this work that would be of interest in the context of modelling powder snow avalanches include: the use of a level set method to advance the solution beyond the self-intersection time and overcome the main drawback of the boundary integral method that we have used in this paper; including the effect of an underlying slope; adding feedback between the flow and the speed and strength of the source - this is straightforward to include in the existing numerical method, but a sensible model for this feedback needs to be determined, perhaps based on the work of Louge et al. (2011).

\section{Acknowledgement}

I'd like to thank my colleagues Barbara Turnbull (whose work funded by Royal Academy of Engineering award RB4525 gave rise to this idea for modelling powder 
snow avalanches) and Matt Scase for bringing this problem to my attention, and also for helpful discussions and comments on a draft of this paper.

\section{REFERENCES}

Ablowitz, M.J. \& Fokas, A.S. 1997 Complex Variables: Introduction and Applications. Cambridge Univeristy Press.

Baker, G.R. \& Beale, J.T. 2004 Vortex blob methods applied to interfacial motion. J. Comp. Phys. 196, 233-258.

Baker, G.R., Meiron, D.I. \& Orszag, S.A. 1982 Generalized vortex methods for free-surface flow problems. J. Fluid Mech. 123, 477-501.

BAKeR, G.R. \& PhAm, L.D. 2006 A comparison of blob methods for vortex sheet roll-up. J. Fluid Mech. 547, 297-316.

Batchelor, G.K. 1967 An Introduction to Fluid Dynamics. Cambridge Univeristy Press.

Billingham, J., Needham, D. J., Korsukova, E. \& Munro, R. J. 2017 The initial development of a jet caused by fluid, body and free surface interaction. Part 5. Parasitic capillary waves on an initially horizontal surface. J. Fluid Mech. 836, 850-872.

Caroll, C.S., Turnbull, B. \& Louge, M.Y. 2012 Role of fluid density in shaping eruption currents driven by frontal particle blow-out. Phys. Fluids 24, 066603.

Cowley, S.J., Baker, G.R. \& TAnveer, S. 1999 On the formation of Moore curvature singularities in vortex sheets. J. Fluid Mech. 378, 233-268.

Ebin, G. 1988 Ill-posedness of the Rayleigh-Taylor and Kelvin-Helmholz problems for incompressible fluids. Comm. Part. Diff. Eqns 13, 1265-1295.

Hou, T.Y., Lowengrub, J.S. \& Shelley, M.J. 1994 Removing the stiffness from interfacial flows with surface tension. J. Comp.Phys. 114, 312-338.

King, A.C. \& Needham, D.J. 1994 The initial development of a jet caused by fluid, body and free-surface interaction. Part 1. A uniformly accelerating plate. J. Fluid Mech. 268, 89-101.

Korobkin, A. \& Yilmaz, O. 2009 The initial stage of dam-break flow. J. Eng. Math. 63, 293-308.

Longuet-Higgins, M.S. \& Cokelet, E.D. 1976 The deformation of steep surface waves on water - i. a numerical method of computation. Proc. R. Soc. Lond. A 350 (1660), 1-26.

Louge, M. Y., Carroll, C. S. \& Turnbull, B. 2011 Role of pore pressure gradients in sustaining frontal particle entrainment in eruption currents: The case of powder snow avalanches. Journal of Geophysical Research: Earth Surface 116 (F4).

Moore, D.W. 1978 The equation of motion of a vortex layer of small thickness. Stud. Appl. Math. 58, 119-140.

Needham, D.J., Chamberlain, P.G. \& Billingham, J. 2008 The initial development of a jet caused by fluid, body and free-surface interaction. Part 3 . An inclined accelerating plate. Q. J. Mech. Appl. Math. 61, 581-614.

Sovilla, B., Burlando, P. \& Bartelt, P. 2006 Field experiments and numerical modeling of mass entrainment in snow avalanches. Journal of Geophysical Research: Earth Surface 111 (F3).

Uddin, J. \& Needham, D.J. 2015 The effects of surface tension on the initial development of a free surface adjacent to an accelerated plate. J. Fluid Mech. 776, 37-73. 